\title{
GROUND, SYMMETRIC AND CENTRAL VORTEX STATES IN ROTATING BOSE-EINSTEIN CONDENSATES *
}

\author{
WEIZHU BAO ${ }^{\dagger}$, HANQUAN WANG ${ }^{\ddagger}$, AND PETER A. MARKOWICH $\S$
}

\begin{abstract}
We study ground, symmetric and central vortex states, as well as their energy and chemical potential diagrams, in rotating Bose-Einstein condensates (BEC) analytically and numerically. We start from the three-dimensional (3D) Gross-Pitaevskii equation (GPE) with an angular momentum rotation term, scale it to obtain a four-parameter model, reduce it to a $2 \mathrm{D}$ GPE in the limiting regime of strong anisotropic confinement and present its semiclassical scaling and geometrical optics. We discuss the existence/nonexistence problem for ground states (depending on the angular velocity) and find that symmetric and central vortex states are independent of the angular rotational momentum. We perform numerical experiments computing these states using a continuous normalized gradient flow (CNGF) method with a backward Euler finite difference (BEFD) discretization. Ground, symmetric and central vortex states, as well as their energy configurations, are reported in $2 \mathrm{D}$ and $3 \mathrm{D}$ for a rotating BEC. Through our numerical study, we find various configurations with several vortices in both $2 \mathrm{D}$ and $3 \mathrm{D}$ structures, energy asymptotics in some limiting regimes and ratios between energies of different states in a strong replusive interaction regime. Finally we report the critical angular velocity at which the ground state loses symmetry, numerical verification of dimension reduction from $3 \mathrm{D}$ to $2 \mathrm{D}$, errors for the Thomas-Fermi approximation, and spourous numerical ground states when the rotation speed is larger than the minimal trapping frequency in the $x y$ plane.
\end{abstract}

Key words. rotating Bose-Einstein condensate, Gross-Pitaevskii equation, ground state, symmetric state, central vortex state, angular momentum rotation, continuous normalized gradient flow, energy, chemical potential.

AMS subject classifications. 35B40, 35P30, 35Q55, 65N25, 81Q05.

\section{Introduction}

Since its realization in dilute bosonic atomic gases $[4,10]$, Bose-Einstein condensation (BEC) of alkali atoms and hydrogen has been produced and studied extensively in the laboratory $[34,38]$, and has permitted an intriguing glimpse into the macroscopic quantum world. In view of potential applications [22], the study of quantized vortices, which are related to superfluid properties, is one of the key issues. In fact, bulk superfluids are distinguished from normal fluids by their ability to support dissipationless flow. Such persistent currents are intimately related to the existence of quantized vortices, which are localized phase singularities with integer topological charge [22]. The superfluid vortex is an example of a topological defect that is well known in superconductors [30] and in liquid helium [18]. The occurrence of quantized vortices in superfluids has been the focus of fundamental theoretical and experimental work [18]. Different groups have obtained quantized vortices in a BEC experimentally, e.g. the JILA group [34], the ENS group [33, 39] and the MIT group [38]. Currently, there are at least two typical ways to generate quantized vortices from the BEC ground state: (i) impose a laser beam rotating with an angular velocity on the magnetic trap holding the atoms to create a harmonic anisotropic potential

*Received: May 10, 2004; accepted (in revised version): January 27, 2005. Communicated by Jack X. Xin.

${ }^{\dagger}$ Department of Computational Science, National University of Singapore, Singapore 117543, Fax: 65-67746756, URL: http://www.cz3.nus.edu.sg/ bao/, (bao@cz3.nus.edu.sg).

${ }^{\ddagger}$ Department of Computational Science, National University of Singapore, Singapore 117543, (wanghanq@cz3.nus.edu.sg).

§Institut für Mathematik, Universität Wien, Nordbergstr. 15,1090 Vienna, Austria, http://mailbox.univie.ac.at/peter.markowich, (peter.markowich@univie.ac.at). 
[42], (ii) add to the stationary magnetic trap a narrow, moving Gaussian potential, representing a far-blue-detuned laser [28]. In fact, recent experimental and theoretical advances in exploration of quantized vortices in a BEC have spurred great excitement in the atomic physics community and renewed interest in studying superfluidity.

The properties of a BEC in a rotational frame at temperatures $T$ much smaller than the critical condensation temperature $T_{c}$ are usually well modelled by a nonlinear Schrödinger equation (NLSE) for the macroscopic wave function known as the Gross-Pitaevskii equation (GPE) [36], which incorporates the trap potential, rotational frame, as well as the interactions among the atoms. The effect of the interactions is described by a mean field which leads to a nonlinear term in the GPE. The cases of repulsive and attractive interactions - which can both be realized in the experiment - correspond to defocusing and focusing nonlinearities in the GPE, respectively.

There has been a series of recent numerical studies for the stability and dynamics of quantized vortices in BEC. For a non-rotating BEC, Bao and Du [6] presented a CNGF with BEFD discretization to compute central vortex states, Caradoc-Davis et. al $[12,13]$, Bao and Zhang [9] studied stability of central vortices and their interactions numerically, Lundh et. al [32] studied free expansion of vortex state. For a rotating $\mathrm{BEC}$, in the line of adding a far-blue-detuned Gaussian laser stirrer, Caradoc-Davis et. al [12, 13], Jackson et. al [27, 28], and Bao et. al [7, 9] studied dynamics of vortices, generation of vortices from the ground state and critical angular speed; in the line of a BEC in a rotational frame, Aftalion and Du [1], Aftalion and Riviere [2] studied numerically and asymptotically ground state, critical angular velocity and energy diagram in the Thomas-Fermi (TF) or semiclassical regime, Aftalion and Danaila [3] and Modugno et. al [35] reported bent vortices, e.g. S-shaped vortex and U-shaped vortex, numerically in cigar-shaped condensation and compared with experimental results [39], Garcia-Ripoll and Perez-Garcia [24, 23, 26] studied stability of the central vortex, Tsubota et. al [45] reported vortex lattice formation. Moreover, Svidzinsky and Fetter [42] have studied dynamics of a vortex line depending on its curvature. For an analysis of the GP-functional in a rotational frame we refer to [40]. For a numerical and theoretical review of quantized vortices, we refer to [22] and the recent book [37].

The aim of this paper is to analytically and numerically study ground, symmetric and central vortex states, as well as their energy diagrams in a BEC under a rotational frame representing a laser beam rotating with a given angular velocity on the magnetic trap. We extend the efficient and stable numerical method of CNGF with BEFD discretization, proposed in [6] for computing ground state of a non-rotating $\mathrm{BEC}$, to a rotating BEC, and then apply it to study the ground state, symmetric state, central vortex states, central vortex ground state, as well as their energy diagrams, in a rotating BEC numerically. Vortices and energy bifurcation are observed in the ground state when the angular rotation speed is bigger than a critical frequency. These results agree very well with those, obtained by theoretical and other numerical methods, in the physical literatures $[1,25,32,40,41]$. Furthermore, we also present some new analytical and numerical results for the ground, symmetric and central vortex states, as well as their energy diagrams in a rotating BEC. These results are to: (i) provide asymptotics of the energy and chemical potential of the ground state in the semiclassical regime; (ii) show that the ground state is a global minimizer of the energy functional over the unit sphere and all excited states are saddle points in the linear case; (iii) provide semiclassical scaling and geometrical optics for a rotating 
BEC; (iv) find numerically the ratio between energies of different stationary states goes to constant in the semiclassical regime; (v) find the asymptotics of the energy of the ground state when the angular momentum velocity is near the minimal trapping frequency in $x y$ plane; (vi) perform a numerical verification for dimension reduction from $3 \mathrm{D}$ to $2 \mathrm{D}$ and find the convergence rate; (vii) define the central vortex ground state and find the critical rotation speed for it numerically; (viii) study the TF approximation numerically; (ix) test different choices of initial data for the numerical method in an isotropic trap and find there is only one choice which always guarantees convergence to the ground state where it exists.

The paper is organized as follows. In section 2, we take the 3D GPE with an angular momentum term, scale it to get a four parameter model, reduce it to a $2 \mathrm{D}$ problem in a limiting regime, and present its semiclassical scaling and geometrical optics. In section 3, we study the energy functional and (for the sake of completeness and readability) present a simple proof of existence of the ground state when the angular speed is less than the minimal trapping frequency in the $x y$ plane, and resp., nonexistence of the ground state when the angular speed is bigger than the maximal trapping frequency in the $x y$ plane. In section 4 , we present symmetric, central vortex and central vortex ground states. In section 5, we extend the CNGF and its BEFD discretization, proposed for non-rotating BEC in [6], to rotating BEC for computing ground and vortex states. In section 6 , we report numerical results in $2 \mathrm{D}$ and $3 \mathrm{D}$, and finally in section 7 we draw some conclusions.

\section{GPE in a rotational frame}

At temperatures $T$ much smaller than the critical temperature $T_{c}$ [30], a BEC in a rotational frame is well described by the macroscopic wave function $\psi(\mathbf{x}, t)$, whose evolution is governed by a self-consistent, mean field nonlinear Schrödinger equation known as the Gross-Pitaevskii equation (GPE) with an angular momentum rotational term $[1,11,20,24]$, (w.l.o.g.) assuming the rotation being around the $z$-axis:

$$
\begin{aligned}
i \hbar \frac{\partial \psi(\mathbf{x}, t)}{\partial t} & =\frac{\delta E(\psi)}{\delta \psi^{*}}:=H \psi \\
& =\left(-\frac{\hbar^{2}}{2 m} \nabla^{2}+V(\mathbf{x})+N U_{0}|\psi(\mathbf{x}, t)|^{2}-\Omega L_{z}\right) \psi(\mathbf{x}, t),
\end{aligned}
$$

where $\mathbf{x}=(x, y, z)^{T} \in \mathbb{R}^{3}$ is the spatial coordinate vector, $m$ is the atomic mass, $\hbar$ is the Planck constant, $N$ is the number of atoms in the condensate, $\Omega$ is an angular velocity, $V(\mathbf{x})$ is an external trapping potential. When a harmonic trap potential is considered, $V(\mathbf{x})=\frac{m}{2}\left(\omega_{x}^{2} x^{2}+\omega_{y}^{2} y^{2}+\omega_{z}^{2} z^{2}\right)$ with $\omega_{x}, \omega_{y}$ and $\omega_{z}$ being the trap frequencies in $x$ , $y$ - and $z$-direction respectively. For the following we assume (w.l.o.g.) $\omega_{x} \leq \omega_{y}$. $U_{0}=\frac{4 \pi \hbar^{2} a_{s}}{m}$ describes the interaction between atoms in the condensate with the $s$ wave scattering length $a_{s}$ (positive for repulsive interaction and negative for attractive interaction) and

$$
L_{z}=x p_{y}-y p_{x}=-i \hbar\left(x \partial_{y}-y \partial_{x}\right)
$$

is the $z$-component of the angular momentum $\mathbf{L}=\mathbf{x} \times \mathbf{P}$ with the momentum operator $\mathbf{P}=-i \hbar \nabla=\left(p_{x}, p_{y}, p_{z}\right)^{T}$. The energy functional per particle $E(\psi)$ is defined as

$$
E(\psi)=\int_{\mathbb{R}^{3}}\left[\frac{\hbar^{2}}{2 m}|\nabla \psi|^{2}+V(\mathbf{x})|\psi|^{2}+\frac{N U_{0}}{2}|\psi|^{4}-\Omega \psi^{*} L_{z} \psi\right] d \mathbf{x} .
$$


Here we use $f^{*}$ to denote the conjugate of a function $f$. It is convenient to normalize the wave function by requiring

$$
\int_{\mathbb{R}^{3}}|\psi(\mathbf{x}, t)|^{2} d \mathbf{x}=1
$$

2.1. Dimensionless GPE in a rotational frame. By introducing the dimensionless variables: $t \rightarrow t / \omega_{m}$ with $\omega_{m}=\min \left\{\omega_{x}, \omega_{y}, \omega_{z}\right\}, \mathbf{x} \rightarrow \mathbf{x} a_{0}$ with $a_{0}=$ $\sqrt{\hbar / m \omega_{m}}, \psi \rightarrow \psi / a_{0}^{3 / 2}, \Omega \rightarrow \Omega \omega_{m}$ and $E(\cdot) \rightarrow \hbar \omega_{m} E_{\beta, \Omega}(\cdot)$, we get the dimensionless GPE

$$
\begin{aligned}
i \frac{\partial \psi(\mathbf{x}, t)}{\partial t} & =\frac{\delta E_{\beta, \Omega}(\psi)}{\delta \psi^{*}}:=H \psi \\
& =\left(-\frac{1}{2} \nabla^{2}+V(\mathbf{x})+\beta|\psi(\mathbf{x}, t)|^{2}-\Omega L_{z}\right) \psi(\mathbf{x}, t)
\end{aligned}
$$

where $\beta=\frac{U_{0} N}{a_{0}^{3} \hbar \omega_{m}}=\frac{4 \pi a_{s} N}{a_{0}}, \quad L_{z}=-i\left(x \partial_{y}-y \partial_{x}\right), \quad V(\mathbf{x})=\frac{1}{2}\left(\gamma_{x}^{2} x^{2}+\gamma_{y}^{2} y^{2}+\gamma_{z}^{2} z^{2}\right) \quad$ with $\gamma_{x}=\frac{\omega_{x}}{\omega_{m}}, \gamma_{y}=\frac{\omega_{y}}{\omega_{m}}$ and $\gamma_{z}=\frac{\omega_{z}}{\omega_{m}}$, and the dimensionless energy functional per particle $E_{\beta, \Omega}(\psi)$ is defined as

$$
E_{\beta, \Omega}(\psi)=\int_{\mathbb{R}^{3}}\left[\frac{1}{2}|\nabla \psi(\mathbf{x}, t)|^{2}+V(\mathbf{x})|\psi|^{2}+\frac{\beta}{2}|\psi|^{4}-\Omega \psi^{*} L_{z} \psi\right] d \mathbf{x} .
$$

In a disk-shaped condensation with parameters $\omega_{x} \approx \omega_{y}$ and $\omega_{z} \gg \omega_{x}\left(\Longleftrightarrow \gamma_{x}=1\right.$, $\gamma_{y} \approx 1$ and $\gamma_{z} \gg 1$ with choosing $\left.\omega_{m}=\omega_{x}\right)$, the 3D GPE $(2.5)$ can be reduced to a $2 \mathrm{D}$ GPE with $\mathbf{x}=(x, y)^{T}[7,5,8]$ :

$$
i \frac{\partial \psi(\mathbf{x}, t)}{\partial t}=-\frac{1}{2} \nabla^{2} \psi+V_{2}(x, y) \psi+\beta_{2}|\psi|^{2} \psi-\Omega L_{z} \psi
$$

where $\beta_{2} \approx \beta_{2}^{a}=\beta \sqrt{\gamma_{z} / 2 \pi}$ and $V_{2}(x, y)=\frac{1}{2}\left(\gamma_{x}^{2} x^{2}+\gamma_{y}^{2} y^{2}\right)[1,7,8]$. Thus here we consider the dimensionless GPE in a rotational frame in $d$-dimensions $(d=2,3)$ :

$$
i \frac{\partial \psi(\mathbf{x}, t)}{\partial t}=-\frac{1}{2} \nabla^{2} \psi+V_{d}(\mathbf{x}) \psi+\beta_{d}|\psi|^{2} \psi-\Omega L_{z} \psi, \quad \mathbf{x} \in \mathbb{R}^{d}, \quad t \geq 0
$$

where $\beta_{3}=\beta$ and $V_{3}(x, y, z)=V(x, y, z)$.

Two important invariants of (2.8) are the normalization of the wave function

$$
N(\psi)=\int_{\mathbb{R}^{d}}|\psi(\mathbf{x}, t)|^{2} d \mathbf{x} \equiv \int_{\mathbb{R}^{d}}|\psi(\mathbf{x}, 0)|^{2} d \mathbf{x}=1, \quad t \geq 0
$$

and the energy

$$
E_{\beta, \Omega}(\psi)=\int_{\mathbb{R}^{d}}\left[\frac{1}{2}|\nabla \psi(\mathbf{x}, t)|^{2}+V_{d}(\mathbf{x})|\psi|^{2}+\frac{\beta_{d}}{2}|\psi|^{4}-\Omega \psi^{*} L_{z} \psi\right] d \mathbf{x} .
$$

2.2. Stationary states. To find a stationary solution of (2.8), we write

$$
\psi(\mathbf{x}, t)=e^{-i \mu t} \phi(\mathbf{x})
$$

where $\mu$ is the chemical potential of the condensate and $\phi$ is independent of time. Inserting (2.11) into (2.8) gives the following equation for $\phi(\mathbf{x})$

$$
\mu \phi(\mathbf{x})=-\frac{1}{2} \Delta \phi(\mathbf{x})+V_{d}(\mathbf{x}) \phi(\mathbf{x})+\beta_{d}|\phi(\mathbf{x})|^{2} \phi(\mathbf{x})-\Omega L_{z} \phi(\mathbf{x}), \quad \mathbf{x} \in \mathbb{R}^{d}
$$


under the normalization condition

$$
\|\phi\|^{2}=\int_{\mathbb{R}^{d}}|\phi(\mathbf{x})|^{2} d \mathbf{x}=1 .
$$

This is a nonlinear eigenvalue problem with a constraint and any eigenvalue $\mu$ can be computed from its corresponding eigenfunction $\phi$ by

$$
\begin{aligned}
\mu & =\mu_{\beta, \Omega}(\phi)=\int_{\mathbb{R}^{d}}\left[\frac{1}{2}|\nabla \phi(\mathbf{x})|^{2}+V_{d}(\mathbf{x})|\phi(\mathbf{x})|^{2}+\beta_{d}|\phi(\mathbf{x})|^{4}-\Omega \phi^{*}(\mathbf{x}) L_{z} \phi(\mathbf{x})\right] d \mathbf{x} \\
& =E_{\beta, \Omega}(\phi)+\int_{\mathbb{R}^{d}} \frac{\beta_{d}}{2}|\phi(\mathbf{x})|^{4} d \mathbf{x} .
\end{aligned}
$$

In fact, the eigenfunctions of (2.12) under the constraint (2.13) are the critical points of the energy functional $E_{\beta, \Omega}(\phi)$ over the unit sphere $S=\left\{\phi \in \mathbb{C} \mid\|\phi\|=1, E_{\beta, \Omega}(\phi)<\infty\right\}$. Furthermore (2.12) is the Euler-Lagrange equation of the energy functional (2.10) with $\psi=\phi$ under the constraint (2.13).

2.3. Semiclassical scaling and geometrical optics. When $\beta_{d} \gg 1$, i.e. in a strongly repulsive interacting condensation or in a semiclassical regime, another scaling (under the normalization (2.9) with $\psi=\psi^{\varepsilon}$ ) for the GPE (2.8) is also very useful in practice by choosing $\mathbf{x} \rightarrow \varepsilon^{-1 / 2} \mathbf{x}$ and $\psi=\psi^{\varepsilon} \varepsilon^{d / 4}$ with $\varepsilon=\beta_{d}^{-2 /(d+2)}$ :

$$
\begin{aligned}
i \varepsilon \frac{\partial \psi^{\varepsilon}(\mathbf{x}, t)}{\partial t} & =\frac{\delta E_{\varepsilon, \Omega}\left(\psi^{\varepsilon}\right)}{\delta\left(\psi^{\varepsilon}\right)^{*}}:=H^{\varepsilon} \psi^{\varepsilon} \\
& =-\frac{\varepsilon^{2}}{2} \nabla^{2} \psi^{\varepsilon}+V_{d}(\mathbf{x}) \psi^{\varepsilon}+\left|\psi^{\varepsilon}\right|^{2} \psi^{\varepsilon}-\varepsilon \Omega L_{z} \psi^{\varepsilon}, \quad \mathbf{x} \in \mathbb{R}^{d},
\end{aligned}
$$

where the energy functional $E_{\varepsilon, \Omega}\left(\psi^{\varepsilon}\right)$ is defined as

$$
E_{\varepsilon, \Omega}\left(\psi^{\varepsilon}\right)=\int_{\mathbb{R}^{3}}\left[\frac{\varepsilon^{2}}{2}\left|\nabla \psi^{\varepsilon}\right|^{2}+V_{d}(\mathbf{x})\left|\psi^{\varepsilon}\right|^{2}+\frac{1}{2}\left|\psi^{\varepsilon}\right|^{4}-\varepsilon \Omega\left(\psi^{\varepsilon}\right)^{*} L_{z} \psi^{\varepsilon}\right] d \mathbf{x}=O(1),
$$

assuming that $\psi^{\varepsilon}$ is $\varepsilon$-oscillatory and 'sufficiently' integrable such that all terms have $O(1)$-integral. Similarly, the nonlinear eigenvalue problem (2.12) (under the normalization (2.13) with $\phi=\phi^{\varepsilon}$ ) reads

$$
\mu^{\varepsilon} \phi^{\varepsilon}(\mathbf{x})=-\frac{\varepsilon^{2}}{2} \Delta \phi^{\varepsilon}+V_{d}(\mathbf{x}) \phi^{\varepsilon}+\left|\phi^{\varepsilon}\right|^{2} \phi^{\varepsilon}-\varepsilon \Omega L_{z} \phi^{\varepsilon}, \quad \mathbf{x} \in \mathbb{R}^{d},
$$

where any eigenvalue $\mu^{\varepsilon}$ can be computed from its corresponding eigenfunction $\phi^{\varepsilon}$ by

$$
\mu^{\varepsilon}=\mu_{\varepsilon, \Omega}\left(\phi^{\varepsilon}\right)=\int_{\mathbb{R}^{d}}\left[\frac{\varepsilon^{2}}{2}\left|\nabla \phi^{\varepsilon}\right|^{2}+V_{d}(\mathbf{x})\left|\phi^{\varepsilon}\right|^{2}+\left|\phi^{\varepsilon}\right|^{4}-\varepsilon \Omega\left(\psi^{\varepsilon}\right)^{*} L_{z} \psi^{\varepsilon}\right] d \mathbf{x}=O(1) .
$$

Furthermore it is easy to get the leading asymptotics of the energy functional $E_{\beta, \Omega}(\psi)$ in (2.10) and the chemical potential $\mu_{\beta, \Omega}(\phi)$ in (2.14) when $\beta_{d} \gg 1$ from this scaling:

$$
\begin{aligned}
& E_{\beta, \Omega}(\psi)=\varepsilon^{-1} E_{\varepsilon, \Omega}\left(\psi^{\varepsilon}\right)=O\left(\varepsilon^{-1}\right)=O\left(\beta_{d}^{2 /(d+2)}\right), \\
& \mu_{\beta, \Omega}(\phi)=\varepsilon^{-1} \mu_{\varepsilon, \Omega}\left(\phi^{\varepsilon}\right)=O\left(\varepsilon^{-1}\right)=O\left(\beta_{d}^{2 /(d+2)}\right), \quad \beta_{d} \gg 1 .
\end{aligned}
$$

These asymptotics results will be confirmed by our numerical results in Section 6 . 
When $0<\varepsilon \ll 1$, i.e. $\beta_{d} \gg 1$, we set

$$
\psi^{\varepsilon}(\mathbf{x}, t)=\sqrt{\rho^{\varepsilon}(\mathbf{x}, t)} \exp \left(\frac{i}{\varepsilon} S^{\varepsilon}(\mathbf{x}, t)\right),
$$

where $\rho^{\varepsilon}=\left|\psi^{\varepsilon}\right|^{2}$ and $S^{\varepsilon}$ is the phase of the wave-function. Inserting (2.19) into (2.15) and collecting real and imaginary parts, we get the transport equation for $\rho^{\varepsilon}$ and the Hamilton-Jacobi equation for the phase $S^{\varepsilon}$ :

$$
\begin{aligned}
& \partial_{t} \rho^{\varepsilon}+\operatorname{div}\left(\rho^{\varepsilon} \nabla S^{\varepsilon}\right)+\Omega\left(x \partial_{y}-y \partial_{x}\right) \rho^{\varepsilon}=0, \\
& \partial_{t} S^{\varepsilon}+\frac{1}{2}\left|\nabla S^{\varepsilon}\right|^{2}+V_{d}(\mathbf{x})+\rho^{\varepsilon}+\Omega\left(x \partial_{y}-y \partial_{x}\right) S^{\varepsilon}=\frac{\varepsilon^{2}}{2} \frac{1}{\sqrt{\rho^{\varepsilon}}} \Delta \sqrt{\rho^{\varepsilon}} .
\end{aligned}
$$

\section{Ground state}

The ground state wave function $\phi^{g}(\mathbf{x}):=\phi_{\beta, \Omega}^{g}(\mathbf{x})$ of a rotating BEC is found by minimizing the energy functional $E_{\beta, \Omega}(\phi)$ over the unit sphere $S$ :

(I) Find $\left(\mu_{\beta, \Omega}^{g}, \phi_{\beta, \Omega}^{g} \in S\right)$ such that

$$
E^{g}:=E_{\beta, \Omega}^{g}=E_{\beta, \Omega}\left(\phi_{\beta, \Omega}^{g}\right)=\min _{\phi \in S} E_{\beta, \Omega}(\phi), \quad \mu^{g}:=\mu_{\beta, \Omega}^{g}=\mu_{\beta, \Omega}\left(\phi_{\beta, \Omega}^{g}\right) .
$$

Any eigenfunction $\phi(\mathbf{x})$ of (2.12) under the constraint (2.13) whose energy $E_{\beta, \Omega}(\phi)>$ $E_{\beta, \Omega}\left(\phi_{\beta, \Omega}^{g}\right)$ is usually called as an excited state in the physical literature [37].

Existence/nonexistence results of ground state, depending on the magnitude $|\Omega|$ of the angular velocity relative to the trapping frequencies are known and can be found [40]. For the sake of readability of this paper we include the sketch of the proof here.

3.1. Existence of the ground state when $|\Omega|<\gamma_{x y}:=\min \left\{\gamma_{x}, \gamma_{y}\right\}$. To study the existence of the ground state in a rotating BEC, we first present some properties of the energy functional

LEMMA 3.1. i) In 2D, we have

$$
E_{\beta,-\Omega}(\phi(x,-y))=E_{\beta, \Omega}(\phi(x, y)), \quad E_{\beta,-\Omega}(\phi(-x, y))=E_{\beta, \Omega}(\phi(x, y)), \quad \phi \in S .
$$

ii) In 3D, we have

$$
E_{\beta,-\Omega}(\phi(x,-y, z))=E_{\beta, \Omega}(\phi(x, y, z)), E_{\beta,-\Omega}(\phi(-x, y, z))=E_{\beta, \Omega}(\phi(x, y, z)), \phi \in S .
$$

iii) In $2 D$ and $3 D$, we have

$$
\begin{aligned}
& \int_{\mathbb{R}^{d}}\left[\frac{1-|\Omega|}{2}|\nabla \phi(\mathbf{x})|^{2}+\left(V_{d}(\mathbf{x})-\frac{|\Omega|}{2}\left(x^{2}+y^{2}\right)\right)|\phi|^{2}+\frac{\beta_{d}}{2}|\phi|^{4}\right] d \mathbf{x} \leq E_{\beta, \Omega}(\phi) \\
& \leq \int_{\mathbb{R}^{d}}\left[\frac{1+|\Omega|}{2}|\nabla \phi(\mathbf{x})|^{2}+\left(V_{d}(\mathbf{x})+\frac{|\Omega|}{2}\left(x^{2}+y^{2}\right)\right)|\phi|^{2}+\frac{\beta_{d}}{2}|\phi|^{4}\right] d \mathbf{x} .
\end{aligned}
$$


Proof. i) From (2.10) with $\psi=\phi$ and $d=2$, observing (2.2), with a change of variables, we have

$$
\begin{aligned}
& E_{\beta,-\Omega}(\phi(x,-y))=\int_{\mathbb{R}^{2}}\left[\frac{1}{2}|\nabla \phi(x,-y)|^{2}+V_{d}(\mathbf{x})|\phi(x,-y)|^{2}\right. \\
& \left.+\frac{\beta_{d}}{2}|\phi(x,-y)|^{4}+\Omega \phi^{*}(x,-y) L_{z} \phi(x,-y)\right] d x d y \\
& \stackrel{y \rightarrow-y}{=} \int_{\mathbb{R}^{2}}\left[\frac{1}{2}|\nabla \phi(x, y)|^{2}+V_{d}(\mathbf{x})|\phi(x, y)|^{2}\right. \\
& \left.+\frac{\beta_{d}}{2}|\phi(x, y)|^{4}-\Omega \phi^{*}(x, y) L_{z} \phi(x, y)\right] d x d y \\
& =E_{\beta, \Omega}(\phi(x, y)), \quad \phi \in S .
\end{aligned}
$$

Similarly, we obtain the second equality in (3.2).

ii) The proof is similar as in i) except $d=3$, details are omitted.

iii) From (2.2), the Hölder inequality, we have

$$
\begin{aligned}
& \left|\int_{\mathbb{R}^{d}}-\Omega \phi^{*}(\mathbf{x}) L_{z} \phi(\mathbf{x}) d \mathbf{x}\right| \leq|\Omega| \int_{\mathbb{R}^{d}}\left|\phi^{*}(\mathbf{x}) L_{z} \phi(\mathbf{x})\right| d \mathbf{x} \\
& =|\Omega| \int_{\mathbb{R}^{d}}\left|\phi^{*}(\mathbf{x})\left(x \partial_{y} \phi-y \partial_{x} \phi\right)\right| d \mathbf{x} \leq|\Omega| \int_{\mathbb{R}^{d}}\left[\left|x \phi^{*}\right|\left|\partial_{y} \phi\right|+\left|y \phi^{*}\right|\left|\partial_{x} \phi\right|\right] d \mathbf{x} \\
& \leq \frac{|\Omega|}{2} \int_{\mathbb{R}^{d}}\left[\left(\left|\partial_{x} \phi\right|^{2}+\left|\partial_{y} \phi\right|^{2}\right)+\left(x^{2}+y^{2}\right)|\phi|^{2}\right] d \mathbf{x} .
\end{aligned}
$$

Thus the inequality (3.4) is a combination of (3.6) and (2.10) with $\psi=\phi$.

From this lemma, since $\gamma_{y} \geq \gamma_{x}=\gamma_{x y}$ and $\gamma_{z}>0$, when $\beta_{d} \geq 0$ and $|\Omega|<\gamma_{x y}$, we know that the energy functional $E_{\beta, \Omega}(\phi)$ is positive, coercive and weakly lower semicontinuous on $S$. Thus the existence of a minimum follows from the standard theory [43] and we have

THEOREM 3.2. i) In $2 D$, if $\phi_{\beta, \Omega}(x, y) \in S$ is a ground state of the energy functional $E_{\beta, \Omega}(\phi)$, then $\phi_{\beta, \Omega}(x,-y) \in S$ and $\phi_{\beta, \Omega}(-x, y) \in S$ are ground states of the energy functional $E_{\beta,-\Omega}(\phi)$. Furthermore

$$
E_{\beta, \Omega}^{g}=E_{\beta,-\Omega}^{g}, \quad \mu_{\beta, \Omega}^{g}=\mu_{\beta,-\Omega}^{g} .
$$

ii) In $3 D$, if $\phi_{\beta, \Omega}(x, y, z) \in S$ is a ground state of the energy functional $E_{\beta, \Omega}(\phi)$, then $\phi_{\beta, \Omega}(x,-y, z) \in S$ and $\phi_{\beta, \Omega}(-x, y, z) \in S$ are ground states of the energy functional $E_{\beta,-\Omega}(\phi)$, and (3.7) is also valid.

iii). When $\beta_{d} \geq 0$ and $|\Omega|<\gamma_{x y}$, there exists a minimizer for the minimization problem (3.1), i.e. there exists a ground state.

For understanding the uniqueness question, note that $E_{\beta, \Omega}\left(\alpha \phi_{\beta, \Omega}^{g}\right)=E_{\beta, \Omega}\left(\phi_{\beta, \Omega}^{g}\right)$ for all $\alpha \in \mathbb{C}$ with $|\alpha|=1$. Thus an additional constraint has to be introduced to show uniqueness. For a non-rotating BEC, i.e. $\Omega=0$, the unique positive minimizer is usually taken as the ground state. In fact, the ground state is unique up to a constant $\alpha$ with $|\alpha|=1$, i.e. density of the ground state is unique, when $\Omega=0$. For a rotating BEC under $|\Omega|<\gamma_{x y}$, in section 5, we present a CNGF and its BEFD discretization for 
computing a minimizer of the minimization problem (3.1) with appropriately chosen initial data. From our numerical results, the density of the ground state may no longer be unique when $|\Omega|>\Omega^{c}$ with $\Omega^{c}$ a critical angular rotation speed.

3.2. Nonexistence of ground states when $|\Omega|>\gamma^{x y}:=\max \left\{\gamma_{x}, \gamma_{y}\right\}$. Denote $\gamma_{r}:=\gamma^{x y}$ and notice $\frac{1}{2}\left(\gamma_{x}^{2} x^{2}+\gamma_{y}^{2} y^{2}\right) \leq \frac{1}{2} \gamma_{r}^{2} r^{2}$ with $r=\sqrt{x^{2}+y^{2}}$, we have

$$
\begin{gathered}
E_{\beta, \Omega}(\phi) \leq \frac{1}{2} \int_{0}^{2 \pi} \int_{0}^{\infty}\left[\left|\partial_{r} \phi\right|^{2}+\frac{1}{r^{2}}\left|\partial_{\theta} \phi\right|^{2}+\gamma_{r}^{2} r^{2}|\phi|^{2}+\beta_{2}|\phi|^{4}+2 i \Omega \phi^{*} \partial_{\theta} \phi\right] r d r d \theta \\
E_{\beta, \Omega}(\phi) \leq \frac{1}{2} \int_{-\infty}^{\infty} \int_{0}^{2 \pi} \int_{0}^{\infty}\left[\left|\partial_{r} \phi\right|^{2}+\frac{1}{r^{2}}\left|\partial_{\theta} \phi\right|^{2}+\left|\partial_{z} \phi\right|^{2}+\left(\gamma_{r}^{2} r^{2}+\gamma_{z}^{2} z^{2}\right)|\phi|^{2}\right. \\
\left.+\beta_{2}|\phi|^{4}+2 i \Omega \phi^{*} \partial_{\theta} \phi\right] r d r d \theta d z, \quad d=3
\end{gathered}
$$

where $(r, \theta)$ and $(r, \theta, z)$ are polar (in 2D), and resp., cylindrical coordinates (in 3D). In $2 \mathrm{D}$, let

$$
\phi_{m}(\mathbf{x})=\phi_{m}(r, \theta)=\phi_{m}(r) e^{i m \theta}, \quad \text { with } \phi_{m}(r)=\frac{\gamma_{r}^{(|m|+1) / 2}}{\sqrt{\pi|m| !}} r^{|m|} e^{-\frac{\gamma_{r} r^{2}}{2}}
$$

where $m$ is an integer. In fact, $\phi_{m}(\mathbf{x})$ is the central vortex state with winding number $m$ of the GPE (2.8) with $d=2, \beta_{d}=0$ and $\Omega=0$. It is very easy to check that $\phi_{m}$ satisfies

$$
\begin{aligned}
& \left\|\phi_{m}\right\|=2 \pi \int_{0}^{\infty}\left|\phi_{m}(r)\right|^{2} r d r=1, \quad m \in \mathbb{Z}, \\
& \frac{1}{2}\left[-\frac{1}{r} \frac{d}{d r}\left(r \frac{d}{d r}\right)+r^{2}+\frac{m^{2}}{r^{2}}\right] \phi_{m}(r)=(|m|+1) \gamma_{r} \phi_{m}(r), 0<r<\infty .
\end{aligned}
$$

Thus $\phi_{m} \in S$ and we compute

$$
\begin{aligned}
E_{\beta, \Omega}\left(\phi_{m}(\mathbf{x})\right) & \leq(|m|+1) \gamma_{r}-\Omega m+\beta_{2} \pi \int_{0}^{\infty}\left|\phi_{m}(r)\right|^{4} r d r \\
& =(|m|+1) \gamma_{r}-\Omega m+\frac{\beta_{2} \gamma_{r}(2|m|) !}{4 \pi\left(2^{|m|}(|m| !)\right)^{2}} .
\end{aligned}
$$

Thus when $|\Omega|>\gamma_{r}$, we have

$$
\begin{aligned}
\inf _{\phi \in S} E_{\beta, \Omega}(\phi) & \leq \begin{cases}\lim _{m \rightarrow \infty} E_{\beta, \Omega}\left(\phi_{m}\right) & \Omega>0 \\
\lim _{m \rightarrow \infty} E_{\beta, \Omega}\left(\phi_{-m}\right) & \Omega<0\end{cases} \\
& =\lim _{m \rightarrow \infty}\left(\gamma_{r}-|\Omega|\right)|m|+\gamma_{r}+\frac{\beta_{2} \gamma_{r}(2|m|) !}{4 \pi\left(2^{|m|}(|m| !)\right)^{2}}=-\infty .
\end{aligned}
$$

This implies that there is no minimizer of the minimization problem (3.1) when $|\Omega|>$ $\gamma^{x y}$ in 2D.

Similarly, in 3D, the argument proceeds with the central vortex line state with winding number $m$ of the GPE (2.8) with $d=3, \beta_{d}=0$ and $\Omega=0$

$$
\phi_{m}(\mathbf{x})=\phi_{m}(r, \theta, z)=\phi_{m}(r, z) e^{i m \theta}, \phi_{m}(r, z)=\frac{\gamma_{r}^{(|m|+1) / 2} \gamma_{z}^{1 / 4}}{\pi^{3 / 4} \sqrt{|m| !}} r^{|m|} e^{-\frac{\gamma_{r} r^{2}+\gamma_{z} z^{2}}{2}},
$$


and we conclude that there is no minimizer of the minimization problem (3.1) when $|\Omega|>\gamma^{x y}$ in $3 \mathrm{D}$.

REMARK 3.1. When $\gamma_{x y}<|\Omega| \leq \gamma^{x y}$ in an anisotropic trap, although there is no rigorous mathematical justification, our numerical results in Section 6 show that there is no ground state of the energy functional $E_{\beta, \Omega}(\phi)$.

3.3. Stationary states as minimizer/saddle points in the linear case. For the stationary states of (2.12), we have the following lemma, valid in the linear case $\beta_{d}=0$ :

Lemma 3.3. Suppose $\beta_{d}=0,|\Omega|<\gamma_{x y}$ and $V_{d}(\mathbf{x}) \geq 0$ for $\mathbf{x} \in \mathbb{R}^{d}$, we have

(i). The ground state $\phi^{g}$ is a global minimizer of $E_{0, \Omega}(\phi)$ over $S$.

(ii). Any excited state $\phi^{e}$ is a saddle point of $E_{0, \Omega}(\phi)$ over $S$.

Proof. Follows the line of the analogous result for a non-rotating BEC in [9].

3.4. Approximate ground state. When $\beta_{d}=0$ and $\Omega=0$, the ground state solution is given explicitly [8]

$$
\mu_{0,0}^{g}=\frac{1}{2}\left\{\begin{array}{l}
\gamma_{x}+\gamma_{y}, \\
\gamma_{x}+\gamma_{y}+\gamma_{z},
\end{array}, \phi_{0,0}^{g}(\mathbf{x})=\frac{1}{\pi^{d / 4}} \begin{cases}\left(\gamma_{x} \gamma_{y}\right)^{1 / 4} e^{-\frac{\gamma_{x} x^{2}+\gamma_{y} y^{2}}{2}}, & d=2 \\
\left(\gamma_{x} \gamma_{y} \gamma_{z}\right)^{1 / 4} e^{-\frac{\gamma_{x} x^{2}+\gamma_{y} y^{2}+\gamma_{z} z^{2}}{2}}, & d=3\end{cases}\right.
$$

In fact, this solution can be viewed as an approximation of the ground state for a weakly interacting slowly rotating condensate, i.e. $\left|\beta_{d}\right| \ll 1$ and $|\Omega| \approx 0$.

For a condensate with strong repulsive interaction, i.e. $\beta_{d} \gg 1,|\Omega| \approx 0, \gamma_{x}=O(1)$, $\gamma_{y}=O(1)$ and $\gamma_{z}=O(1)$, the ground state can be approximated by the TF approximation in this regime $[1,7,8,9]$ :

$$
\begin{aligned}
& \phi_{\beta}^{\mathrm{TF}}(\mathbf{x})= \begin{cases}\sqrt{\left(\mu_{\beta}^{\mathrm{TF}}-V_{d}(\mathbf{x})\right) / \beta_{d}}, & V_{d}(\mathbf{x})<\mu_{\beta}^{\mathrm{TF}}, \\
0, & \text { otherwise, }\end{cases} \\
& \mu_{\beta}^{\mathrm{TF}}=\frac{1}{2} \begin{cases}\left(4 \beta_{2} \gamma_{x} \gamma_{y} / \pi\right)^{1 / 2} & d=2, \\
\left(15 \beta_{3} \gamma_{x} \gamma_{y} \gamma_{z} / 4 \pi\right)^{2 / 5} & d=3 .\end{cases}
\end{aligned}
$$

Clearly $\phi_{\beta}^{\mathrm{TF}}$ is not differentiable at $V_{d}(\mathbf{x})=\mu_{\beta}^{\mathrm{TF}}$, thus $E_{\beta, \Omega}\left(\phi_{\beta}^{\mathrm{TF}}\right)=\infty$ and $\mu_{\beta, \Omega}\left(\phi_{\beta}^{\mathrm{TF}}\right)=$ $\infty[7,9]$. This shows that one can't use (2.10) to define the energy of the TF approximation (3.16). How to define the energy of the TF approximation is not clear in the literature. Using (2.14), (3.17) and (3.16), following [9] for a non-rotating BEC, here we use the way to define the energy of the TF approximation (3.16) [9]:

$$
E_{\beta, \Omega}^{\mathrm{TF}}=\mu_{\beta, \Omega}^{\mathrm{TF}}-\int_{\mathbb{R}^{d}} \frac{\beta_{d}}{2}\left|\phi_{\beta}^{\mathrm{TF}}(\mathbf{x})\right|^{4} d \mathbf{x}=\frac{d+2}{d+4} \mu_{\beta}^{\mathrm{TF}}, \quad d=2,3 .
$$

Our numerical results in Section 6 show that the TF approximation (3.16) is very accurate for the density of the ground state, except at the vortex vore, when $\beta_{d} \gg 1$ and $|\Omega|<\gamma_{x y}$, and (3.17) and (3.18) converge to the chemical potential and energy respectively only when $|\Omega| \approx 0$, but diverge when $|\Omega|$ is near $\gamma_{x y}$.

\section{Excited states}

In this section, we present symmetric, central vortex states and their energies of rotating BEC in 2D with radial symmetry and 3D with cylindrical symmetry, i.e. $\gamma_{x}=\gamma_{y}$ in (2.8). These states, as well as their stability and interaction, were widely 
studied in non-rotating BEC $[7,12,13,27,28,6,9,5]$. They are also very interesting in rotating $\mathrm{BEC}[11,20,34,38,23,26]$. In fact, they will be used as initial data for studying quantized vortex stability [33, 24, 21], dynamics [35, 44] and interaction in superfluidity [42].

4.1. Central vortex ground state. One interesting excited state is a vortex state whose energy minimizes the energy functional among all $\phi \in S$ which is locally a central vortex near the origin in $2 \mathrm{D}$ and a central vortex line near the $z$-axis in $3 \mathrm{D}$. To be specific, in $2 \mathrm{D}$, let

$$
S_{v}=\left\{\phi(\mathbf{x}) \in \mathbb{C} \mid\|\phi\|=1, E_{\beta, \Omega}(\phi)<\infty, \phi(0,0)=0,(0,0) \text { is a vortex center of } \phi\right\}
$$

and in $3 \mathrm{D}$

$$
\begin{aligned}
& S_{v}=\left\{\phi(\mathbf{x}) \in \mathbb{C} \mid\|\phi\|=1, E_{\beta, \Omega}(\phi)<\infty, \phi(0,0, z)=0,(0,0, z)(z \in \mathbb{R})\right. \\
& \quad \text { is a vortex line of } \phi\} .
\end{aligned}
$$

Clearly $S_{v}$ is a subset of the unit sphere $S$, i.e. $S_{v} \subset S$. Then the central vortex ground state $\phi^{v}(\mathbf{x}):=\phi_{\beta, \Omega}^{v}(\mathbf{x})$ is found by minimizing the energy functional $E_{\beta, \Omega}(\phi)$ over the set $S_{v} \subset S$ :

(II) Find $\left(\mu_{\beta, \Omega}^{v}, \phi_{\beta, \Omega}^{v} \in S_{v}\right)$ such that

$$
E^{v}:=E_{\beta, \Omega}^{v}=E_{\beta, \Omega}\left(\phi_{\beta, \Omega}^{v}\right)=\min _{\phi \in S_{v}} E_{\beta, \Omega}(\phi), \quad \mu^{v}:=\mu_{\beta, \Omega}^{v}=\mu_{\beta, \Omega}\left(\phi_{\beta, \Omega}^{v}\right) .
$$

Similarly as the proof for ground state, we have the following theorem for central vortex ground state:

THEOREM 4.1. i) In 2D, if $\phi_{\beta, \Omega}(x, y) \in S_{v}$ is a central vortex ground state of the energy functional $E_{\beta, \Omega}(\phi)$, then $\phi_{\beta, \Omega}(x,-y) \in S_{v}$ and $\phi_{\beta, \Omega}(-x, y) \in S_{v}$ are central vortex ground states of the energy functional $E_{\beta,-\Omega}(\phi)$. Furthermore

$$
E_{\beta, \Omega}^{v}=E_{\beta,-\Omega}^{v}, \quad \mu_{\beta, \Omega}^{v}=\mu_{\beta,-\Omega}^{v} .
$$

ii) In $3 D$, if $\phi_{\beta, \Omega}(x, y, z) \in S_{v}$ is a central vortex ground state of the energy functional $E_{\beta, \Omega}(\phi)$, then $\phi_{\beta, \Omega}(x,-y, z) \in S_{v}$ and $\phi_{\beta, \Omega}(-x, y, z) \in S_{v}$ are central vortex ground states of the energy functional $E_{\beta,-\Omega}(\phi)$, and (4.2) is also valid.

iii). When $\beta_{d} \geq 0$ and $|\Omega|<\gamma_{x y}$, there exists a minimizer for the minimization problem (4.1), i.e. there exists central vortex ground state.

iv). When $\beta_{d} \geq 0$ and $|\Omega|>\gamma^{x y}$, there exists no central vortex ground state.

The CNGF and BEFD discretization for computing the ground state in section 5 can also be applied to compute central vortex ground state numerically for $\beta_{d} \geq 0$ and $|\Omega|<\gamma_{x y}$ provided that we choose appropriate initial data for them.

4.2. Symmetric and central vortex states. In $2 \mathrm{D}$ with radially symmetric trap, i.e. $d=2$ and $\gamma_{y}=\gamma_{x}:=\gamma_{r}$ in (2.8), we consider wave function of the form

$$
\psi(\mathbf{x}, t)=e^{-i \mu_{m} t} \phi_{m}(x, y)=e^{-i \mu_{m} t} \phi_{m}(r) e^{i m \theta}
$$

where $m$ is an integer which corresponds to a symmetric state when $m=0$ with $\phi_{0}^{\prime}(0)=$ 0 and to central vortex states when $m \neq 0$ ( $m$ is usually called index or winding number in this case) with $\phi_{m}(0)=0, \mu_{m}$ is the chemical potential, and $\phi_{m}(r)$ is a real 
function independent of time $t$ and angle $\theta=\operatorname{Arg}(\mathbf{x})$. Inserting (4.3) into (2.8) gives the following equation for $\phi_{m}(r)$ with $0<r<\infty$ :

$$
\begin{aligned}
& \mu_{m} \phi_{m}(r)=\frac{1}{2}\left[-\frac{1}{r} \frac{d}{d r}\left(r \frac{d}{d r}\right)+\gamma_{r}^{2} r^{2}+\frac{m^{2}}{r^{2}}+2 \beta_{2}\left|\phi_{m}\right|^{2}-2 m \Omega\right] \phi_{m}, \\
& \phi_{m}^{\prime}(0)=0 \quad(m=0), \quad \text { and resp. } \quad \phi_{m}(0)=0 \quad(m \neq 0), \\
& \lim _{r \rightarrow \infty} \phi_{m}(r)=0,
\end{aligned}
$$

under the normalization condition

$$
2 \pi \int_{0}^{\infty}\left|\phi_{m}(r)\right|^{2} r d r=1 .
$$

In order to find the radially symmetric state $(m=0)$, and resp., central vortex states with index $m(m \neq 0), \phi_{\beta, \Omega}^{m}(x, y)=\phi_{\beta, \Omega}^{m}(r) e^{i m \theta}$, we need to find a real nonnegative function $\phi^{m}(r):=\phi_{\beta, \Omega}^{m}(r)$ which minimizes the energy functional

$$
\begin{aligned}
& E_{\beta, \Omega}^{m}(\phi(r))=E_{\beta, \Omega}\left(\phi(r) e^{i m \theta}\right) \\
& =\pi \int_{0}^{\infty}\left[\left|\phi^{\prime}(r)\right|^{2}+\left(\gamma_{r}^{2} r^{2}+\frac{m^{2}}{r^{2}}\right)|\phi(r)|^{2}+\beta_{2}|\phi(r)|^{4}-2 m \Omega|\phi(r)|^{2}\right] r d r \\
& =E_{\beta, 0}^{m}(\phi(r))-m \Omega, \quad \Omega \in \mathbb{R},
\end{aligned}
$$

over the set $S_{0}=\left\{\left.\phi(r) \in \mathbb{R}\left|2 \pi \int_{0}^{\infty}\right| \phi(r)\right|^{2} r d r=1, \quad E_{\beta, 0}^{m}(\phi)<\infty, \quad \phi^{\prime}(0)=0 \quad(m=\right.$ $0)$, and resp. $\phi(0)=0(m \neq 0)\}$. The existence and uniqueness of a nonnegative minimizer for this minimization problem can be obtained similarly as for the ground state when $\Omega=0$ [31]. Note that the set $S_{m}=\left\{\phi(r) e^{i m \theta} \mid \phi \in S_{0}\right\} \subset S$ is a subset of the unit sphere, so $\phi_{\beta, \Omega}^{m}(r) e^{i m \theta}$ is a minimizer of the energy functional $E_{\beta, \Omega}(\phi)$ over the set $S_{m} \subset S$. When $\beta_{2}=0$ and $\Omega=0$ in $(2.8), \phi_{0,0}^{m}(r)=\frac{\gamma_{r}^{(|m|+1) / 2}}{\sqrt{\pi|m| !}} r^{|m|} e^{-\gamma_{r} r^{2} / 2}[6]$.

Similarly, in order to find the cylindrically symmetric state $(m=0)$, and resp. central vortex line states $(m \neq 0)$, in 3D with cylindrical symmetry, i.e. $d=3$ and $\gamma_{y}=\gamma_{x}:=\gamma_{r}$ in $(2.8)$, we write

$$
\psi(\mathbf{x}, t)=e^{-i \mu_{m} t} \phi_{m}(x, y, z)=e^{-i \mu_{m} t} \phi_{m}(r, z) e^{i m \theta},
$$

where $m$ is an integer and called as an index when $m \neq 0, \mu_{m}$ is the chemical potential, and $\phi_{m}(r, z)$ is a real function independent of time and angle. Inserting (4.9) into (2.8) with $d=3$ gives the following equation for $\phi_{m}(r, z)$

$$
\begin{aligned}
& \mu_{m} \phi_{m}=\frac{1}{2}\left[-\frac{1}{r} \frac{\partial}{\partial r}\left(r \frac{\partial}{\partial r}\right)-\frac{\partial^{2}}{\partial z^{2}}+\gamma_{r}^{2} r^{2}+\frac{m^{2}}{r^{2}}+\gamma_{z}^{2} z^{2}+2 \beta_{3}\left|\phi_{m}\right|^{2}-2 m \Omega\right] \phi_{m}, \\
& \partial_{r} \phi_{m}(0, z)=0(m=0), \quad \text { and resp. } \quad \phi_{m}(0, z)=0(m \neq 0), \quad z \in \mathbb{R}, \\
& \lim _{r \rightarrow \infty} \phi_{m}(r, z)=0, \quad-\infty<z<\infty, \quad \lim _{|z| \rightarrow \infty} \phi(r, z)=0, \quad 0 \leq r<\infty
\end{aligned}
$$

under the normalization condition

$$
2 \pi \int_{0}^{\infty} \int_{-\infty}^{\infty}\left|\phi_{m}(r, z)\right|^{2} r d r d z=1
$$


In order to find the cylindrically symmetric state $(m=0)$, and resp. central vortex line states $(m \neq 0) \phi_{\beta, \Omega}^{m}(x, y, z)=\phi_{\beta, \Omega}^{m}(r, z) e^{i m \theta}$, we compute a real nonnegative function $\phi^{m}(r, z):=\phi_{\beta, \Omega}^{m}(r, z)$ which minimizes the energy functional

$$
\begin{aligned}
& E_{\beta, \Omega}^{m}(\phi(r, z))=E_{\beta, \Omega}\left(\phi(r, z) e^{i m \theta}\right) \\
& =\pi \int_{0}^{\infty} \int_{-\infty}^{\infty}\left[\left|\partial_{r} \phi\right|^{2}+\left|\partial_{z} \phi\right|^{2}+\left(\gamma_{r}^{2} r^{2}+\gamma_{z}^{2} z^{2}+\frac{m^{2}}{r^{2}}-2 m \Omega\right)|\phi|^{2}+\beta_{3}|\phi|^{4}\right] r d r d z \\
& =E_{\beta, 0}^{m}(\phi(r, z))-m \Omega, \quad \Omega \in \mathbb{R},
\end{aligned}
$$

over the set $S_{0}=\left\{\left.\phi(r, z) \in \mathbb{R}\left|2 \pi \int_{0}^{\infty} \int_{-\infty}^{\infty}\right| \phi(r, z)\right|^{2} r d r d z=1, E_{\beta, 0}^{m}(\phi)<\infty, \partial_{r} \phi(0, r)=\right.$ $0(m=0)$, and resp. $\phi(0, z)=0(m \neq 0),-\infty<z<\infty\}$. The existence and uniqueness of a nonnegative minimizer for this minimization problem can be obtained similarly as for the ground state when $\Omega=0$ [31]. Note that the set $S_{m}=\left\{\phi(r, z) e^{i m \theta} \mid \phi \in\right.$ $\left.S_{0}\right\} \subset S$ is a subset of the unit sphere, so $\phi_{\beta, \Omega}^{m}(r, z) e^{i m \theta}$ is a minimizer of the energy functional $E_{\beta, \Omega}(\phi)$ over the set $S_{m} \subset S$. When $\beta_{3}=0$ and $\Omega=0$ in $(2.8), \phi_{0,0}^{m}(r, z)=$ $\frac{\gamma_{r}^{(|m|+1) / 2} \gamma_{z}^{1 / 4}}{\pi^{3 / 4} \sqrt{|m| !}} r^{|m|} e^{-\left(\gamma_{r} r^{2}+\gamma_{z} z^{2}\right) / 2}[6]$.

When $\beta_{d}>0$, the CNGF and its BEFD discretization proposed in [6] for $\Omega=0$ can be applied directly to compute the central vortex states for $\Omega \neq 0$.

From the above discussions, for symmetric states and central vortex states in a rotating BEC, we have

LEMMA 4.2. i) The radially (in 2D) and cylindrically (in 3D) symmetric states, and central vortex states are independent of the angular momentum term, i.e.

$$
\phi_{\beta, \Omega}^{m}=\phi_{\beta, 0}^{m}, \quad \beta_{d} \geq 0, \quad \Omega \in \mathbb{R}, \quad m \in \mathbb{Z} .
$$

ii) The energy and chemical potential are independent of $\Omega$ for the symmetric states, and resp. are changing linearly with respect to $\Omega$ for the central vortex states, i.e.

$$
\begin{aligned}
E^{m}: & =E_{\beta, \Omega}^{m}\left(\phi_{\beta, \Omega}^{m}\right)=E_{\beta, 0}^{m}\left(\phi_{\beta, 0}^{m}\right)-m \Omega, \\
\mu^{m}: & =\mu_{\beta, \Omega}^{m}\left(\phi_{\beta, \Omega}^{m}\right)=\mu_{\beta, 0}^{m}\left(\phi_{\beta, 0}^{m}\right)-m \Omega, \quad \beta_{d} \geq 0, \quad \Omega \in \mathbb{R}, \quad m \in \mathbb{Z} .
\end{aligned}
$$

iii) When $\Omega=0 \& \beta_{d} \geq 0$, the symmetric state is the same as the ground state, i.e. $\phi_{\beta, \Omega}^{0}=\phi_{\beta, \Omega}^{g}$, and the central vortex state with index $m=1$ is the same as the central vortex ground state, i.e. $\phi_{\beta, \Omega}^{1}=\phi_{\beta, \Omega}^{v}$.

4.3. Critical angular velocity in symmetric trap. In $2 \mathrm{D}$ with radial symmetry and in $3 \mathrm{D}$ with cylindrical symmetry, for any $\beta_{d} \geq 0$, when $\Omega=0$, the ground state satisfies $\phi_{\beta, 0}^{g}(\mathbf{x})=\phi_{\beta, 0}^{0}(r)$ in $2 \mathrm{D}$ and $\phi_{\beta, 0}^{g}(\mathbf{x})=\phi_{\beta, 0}^{0}(r, z)$ in $3 \mathrm{D}$, i.e. the ground state is symmetric. When $\Omega$ increases to a critical angular velocity, $\Omega_{\beta}^{c}$, defined as

$$
\Omega^{c}:=\Omega_{\beta}^{c}=\max \left\{\Omega \mid E_{\beta, \Omega}\left(\phi_{\beta, \Omega}^{g}\right)=E_{\beta, \Omega}\left(\phi_{\beta, \Omega}^{0}\right)=E_{\beta, 0}\left(\phi_{\beta, 0}^{0}\right)\right\},
$$

the energy of the ground state will be less than that of the symmetric state, i.e. symmetry breaking occurs in the ground state $[40,41] . \Omega_{\beta}^{c}$ is also called as critical angular velocity for symmetry breaking in the ground state.

Similarly, for any $\beta_{d} \geq 0$, when $\Omega=0$, the central vortex ground state satisfies $\phi_{\beta, \Omega}^{v}(\mathbf{x})=\phi_{\beta, 0}^{1}(r) e^{i \theta}$ in $2 \mathrm{D}$ and $\phi_{\beta, \Omega}^{v}(\mathbf{x})=\phi_{\beta, 0}^{1}(r, z) e^{i \theta}$ in $3 \mathrm{D}$. When $\Omega$ increases to a critical angular velocity, $\Omega_{\beta}^{v}$, defined as

$$
\Omega^{v}:=\Omega_{\beta}^{v}=\max \left\{\Omega \mid E_{\beta, \Omega}\left(\phi_{\beta, \Omega}^{v}\right)=E_{\beta, \Omega}^{1}\left(\phi_{\beta, 0}^{1}\right)-\Omega\right\},
$$


the energy of $\phi_{\beta, \Omega}^{v}$ will be less than that of the index 1 central vortex state. We call $\Omega_{\beta}^{v}$ as critical angular velocity for symmetry breaking in the central vortex ground state.

From the above discussions and our numerical computations presented later, we find

$$
\Omega_{0}^{c}=\Omega_{0}^{v}=\gamma_{r}, \quad 0 \leq \Omega_{\beta}^{c}<\Omega_{\beta}^{v} \leq \gamma_{r}, \text { for } \beta_{d}>0 .
$$

\section{Normalized gradient flow and its discretization}

Various algorithms, e.g. imaginary time method [15, 1, 2], Sobolev gradient method [25, 24], finite element approximation [8, 46], iterative method [14] etc., for finding the minimizer of the minimization problem (3.1) have been studied in the literatures. Perhaps one of the more popular techniques for dealing with the normalization constraint (2.13) is through the splitting (or projection) scheme: (i). Apply the steepest decent method to an unconstrained minimization problem; (ii) project the solution back to the unit sphere $S$. This suggests to us to consider gradient flow with discrete normalization (GFDN):

$$
\begin{aligned}
& \phi_{t}=-\frac{\delta E_{\beta, \Omega}(\phi)}{\delta \phi^{*}}=\frac{1}{2} \Delta \phi-V_{d}(\mathbf{x}) \phi-\beta_{d}|\phi|^{2} \phi+\Omega L_{z} \phi, \quad t_{n}<t<t_{n+1}, \\
& \phi\left(\mathbf{x}, t_{n+1}\right) \triangleq \phi\left(\mathbf{x}, t_{n+1}^{+}\right)=\frac{\phi\left(\mathbf{x}, t_{n+1}^{-}\right)}{\left\|\phi\left(\cdot, t_{n+1}^{-}\right)\right\|}, \quad \mathbf{x} \in \mathbb{R}^{d}, \quad n \geq 0 \\
& \phi(\mathbf{x}, 0)=\phi_{0}(\mathbf{x}), \quad \mathbf{x} \in \mathbb{R}^{d \quad \text { with } \quad\left\|\phi_{0}\right\|=1 ;}
\end{aligned}
$$

where $0=t_{0}<t_{1}<t_{2}<\cdots<t_{n}<\cdots$ with $\Delta t_{n}=t_{n+1}-t_{n}>0$ and $k=\max _{n \geq 0} \Delta t_{n}$, and $\phi\left(\mathbf{x}, t_{n}^{ \pm}\right)=\lim _{t \rightarrow t_{n}^{ \pm}} \phi(\mathbf{x}, t)$. In fact, the gradient flow (5.1) can be viewed as applying the steepest descent method to the energy functional $E_{\beta, \Omega}(\phi)$ without constraint and (5.2) then projects the solution back to the unit sphere in order to satisfy the constraint (2.13). From the numerical point of view, the gradient flow (5.1) can be solved via traditional techniques and the normalization of the gradient flow is simply achieved by a projection at the end of each time step.

Let

$$
\tilde{\phi}(\cdot, t)=\frac{\phi(\cdot, t)}{\|\phi(\cdot, t)\|}, \quad t_{n} \leq t \leq t_{n+1}, \quad n \geq 0 .
$$

For the gradient flow (5.1), it is easy to establish the following basic facts:

Lemma 5.1. Suppose $V_{d}(\mathbf{x}) \geq 0$ for all $\mathbf{x} \in \mathbb{R}^{d}, \beta_{d} \geq 0$ and $\left\|\phi_{0}\right\|=1$, then

(i). $\|\phi(\cdot, t)\| \leq\left\|\phi\left(\cdot, t_{n}\right)\right\|=1$ for $t_{n} \leq t<t_{n+1}, n \geq 0$.

(ii). For any $\beta_{d} \geq 0$, and all $t^{\prime}, t$ with $t_{n} \leq t^{\prime}<t<t_{n+1}$ :

$$
E_{\beta, \Omega}(\phi(\cdot, t)) \leq E_{\beta, \Omega}\left(\phi\left(\cdot, t^{\prime}\right)\right), \quad n \geq 0
$$

(iii). For $\beta_{d}=0$,

$$
E_{0, \Omega}(\tilde{\phi}(\cdot, t)) \leq E_{0, \Omega}\left(\tilde{\phi}\left(\cdot, t_{n}\right)\right), \quad t_{n} \leq t \leq t_{n+1}, \quad n \geq 0
$$

Proof. Follows the line of the analogous result for a non-rotating BEC in [6]. From Lemma 5.1, we get immediately 
Theorem 5.2. Suppose $V_{d}(\mathbf{x}) \geq 0$ for all $\mathbf{x} \in \mathbb{R}^{d}$ and $\left\|\phi_{0}\right\|=1$. For $\beta_{d}=0$, GFDN (5.1)-(5.3) is energy diminishing for any time step $k$ and initial data $\phi_{0}$, i.e.

$$
E_{0, \Omega}\left(\phi\left(\cdot, t_{n+1}\right)\right) \leq E_{0, \Omega}\left(\phi\left(\cdot, t_{n}\right)\right) \leq \cdots \leq E_{0, \Omega}(\phi(\cdot, 0))=E_{0, \Omega}\left(\phi_{0}\right), n \geq 0 .
$$

In fact, the normalized step (5.2) is equivalent to solve the following ODE exactly

$$
\begin{aligned}
& \phi_{t}(\mathbf{x}, t)=\mu_{\phi}(t, k) \phi(\mathbf{x}, t), \quad \quad \mathbf{x} \in \mathbb{R}^{d}, \quad t_{n}<t<t_{n+1}, \quad n \geq 0, \\
& \phi\left(\mathbf{x}, t_{n}^{+}\right)=\phi\left(\mathbf{x}, t_{n+1}^{-}\right), \quad \mathbf{x} \in \mathbb{R}^{d} ;
\end{aligned}
$$

where

$$
\mu_{\phi}(t, k) \equiv \mu_{\phi}\left(t_{n+1}, \Delta t_{n}\right)=-\frac{1}{2 \Delta t_{n}} \ln \left\|\phi\left(\cdot, t_{n+1}^{-}\right)\right\|^{2}, \quad t_{n} \leq t \leq t_{n+1} .
$$

Thus the GFDN (5.1)-(5.3) can be viewed as a first-order splitting method for the gradient flow with discontinuous coefficients:

$$
\begin{aligned}
& \phi_{t}=\frac{1}{2} \Delta \phi-V_{d}(\mathbf{x}) \phi-\beta|\phi|^{2} \phi+\Omega L_{z} \phi+\mu_{\phi}(t, k) \phi, \quad \mathbf{x} \in \mathbb{R}^{d}, \quad t \geq 0, \\
& \phi(\mathbf{x}, 0)=\phi_{0}(\mathbf{x}), \quad \mathbf{x} \in \mathbb{R}^{d} \quad \text { with } \quad\left\|\phi_{0}\right\|=1 .
\end{aligned}
$$

Letting $k \rightarrow 0$ and noticing that $\phi\left(\mathbf{x}, t_{n+1}\right)$ on the right hand side of (5.9) is the solution of (5.1) at $t_{n+1}=t+\Delta t_{n}$, we obtain

$$
\begin{aligned}
\mu_{\phi}(t) & :=\lim _{k \rightarrow 0^{+}} \mu_{\phi}(t, k)=\lim _{\Delta t_{n} \rightarrow 0^{+}} \frac{1}{-2 \Delta t_{n}} \ln \left\|\phi\left(\cdot, t_{n+1}^{-}\right)\right\|^{2} \\
& =\lim _{\Delta t_{n} \rightarrow 0^{+}} \frac{1}{-2 \Delta t_{n}} \ln \left\|\phi\left(\cdot,\left(t+\Delta t_{n}\right)^{-}\right)\right\|^{2}=\lim _{\Delta t_{n} \rightarrow 0^{+}} \frac{\left.\frac{d}{d \tau}\|\phi(\cdot, t+\tau)\|^{2}\right|_{\tau=\Delta t_{n}}}{-2\left\|\phi\left(\cdot, t+\Delta t_{n}\right)\right\|^{2}} \\
& =\lim _{\Delta t_{n} \rightarrow 0^{+}} \frac{\mu_{\beta, \Omega}\left(\phi\left(\cdot, t+\Delta t_{n}\right)\right.}{\left\|\phi\left(\cdot, t+\Delta t_{n}\right)\right\|^{2}}=\frac{\mu_{\beta, \Omega}(\phi(\cdot, t))}{\|\phi(\cdot, t)\|^{2}} .
\end{aligned}
$$

This suggests to us to consider the following CNGF:

$$
\begin{aligned}
& \phi_{t}=\frac{1}{2} \Delta \phi-V_{d}(\mathbf{x}) \phi-\beta_{d}|\phi|^{2} \phi+\Omega L_{z} \phi+\mu_{\phi}(t) \phi, \quad \mathbf{x} \in \mathbb{R}^{d}, \quad t \geq 0, \\
& \phi(\mathbf{x}, 0)=\phi_{0}(\mathbf{x}), \quad \mathbf{x} \in \mathbb{R}^{d} \quad \text { with } \quad\left\|\phi_{0}\right\|=1 .
\end{aligned}
$$

In fact, the right hand side of (5.14) is the same as (2.12) if we view $\mu_{\phi}(t)$ as a Lagrange multiplier for the constraint (2.13). Furthermore for the above CNGF, as observed in [6] for a non-rotating BEC, the solution of (5.14) also satisfies the following theorem:

Theorem 5.3. Suppose $V_{d}(\mathbf{x}) \geq 0$ for all $\mathbf{x} \in \mathbb{R}^{d}, \beta_{d} \geq 0$ and $\left\|\phi_{0}\right\|=1$. Then the CNGF (5.14)-(5.15) is normalization conserving and energy diminishing, i.e.

$$
\begin{aligned}
& \|\phi(\cdot, t)\|^{2}=\int_{\mathbb{R}^{d}}|\phi(\mathbf{x}, t)|^{2} d \mathbf{x}=\left\|\phi_{0}\right\|^{2}=1, \quad t \geq 0, \\
& \frac{d}{d t} E_{\beta, \Omega}(\phi)=-2\left\|\phi_{t}(\cdot, t)\right\|^{2} \leq 0, \quad t \geq 0,
\end{aligned}
$$

which in turn implies

$$
E_{\beta, \Omega}\left(\phi\left(\cdot, t_{1}\right)\right) \geq E_{\beta, \Omega}\left(\phi\left(\cdot, t_{2}\right)\right), \quad 0 \leq t_{1} \leq t_{2}<\infty .
$$


Proof. Follows the line of the analogous result for a non-rotating BEC in [6].

From this theorem and the inequality (3.4), we may also get as $t \rightarrow \infty$, when $|\Omega|<\gamma_{x y}, \phi$ approaches to a steady state solution which is a critical point of the energy functional $E_{\beta, \Omega}(\phi)[43,6]$. In a non-rotating BEC, i.e. $\Omega=0$, the unique real valued nonnegative ground state solution $\phi_{g}(\mathbf{x}) \geq 0$ for all $\mathbf{x} \in \mathbb{R}^{d}[31]$ is obtained by choosing a positive initial datum $\phi_{0}(\mathbf{x}) \geq 0$ for $\mathbf{x} \in \mathbb{R}^{d}$, e.g. the ground state solution of linear Schrödinger equation with a harmonic oscillator potential $[8,6]$. For a rotating BEC, e.g. $|\Omega|<\gamma_{x y}$, our numerical results in section 6 suggest that the initial data can be chosen as a linear combination of the ground state and central vortex ground state of (2.8) when $\beta_{d}=0$ and $\Omega=0$, which are given explicitly in sections $3 \& 4$.

With this kind of initial data, the ground state solution $\phi_{\beta, \Omega}^{g}$ and its corresponding chemical potential $\mu_{\beta, \Omega}^{g}$ can be obtained from the steady state solution of the CNGF (5.14)-(5.15), i.e.

$$
\phi^{g}(\mathbf{x}):=\phi_{\beta, \Omega}^{g}(\mathbf{x})=\lim _{t \rightarrow \infty} \phi(\mathbf{x}, t), \quad \mathbf{x} \in \mathbb{R}^{d}, \quad \mu^{g}:=\mu_{\beta, \Omega}^{g}=\mu_{\beta, \Omega}\left(\phi_{\beta, \Omega}^{g}\right) .
$$

Furthermore, when $\gamma_{y}=\gamma_{x}$ in (2.8), our numerical results also suggest that when the initial data is chosen as the central vortex ground state of $(2.8)$ when $\beta_{d}=0$ and $\Omega=0$, the steady state solution of the CNGF (5.14)-(5.15) converges to the central vortex ground state solution $\phi_{\beta, \Omega}^{v}$ as $t \rightarrow \infty$, i.e.

$$
\phi^{v}(\mathbf{x}):=\phi_{\beta, \Omega}^{v}(\mathbf{x})=\lim _{t \rightarrow \infty} \phi(\mathbf{x}, t), \quad \mathbf{x} \in \mathbb{R}^{d}, \quad \mu^{v}:=\mu_{\beta, \Omega}^{v}=\mu_{\beta, \Omega}\left(\phi_{\beta, \Omega}^{v}\right) .
$$

Rigourous mathematical justification for these observations is under further study.

In order to derive a full discretization of the GFDN (5.1)-(5.3) (or CNGF (5.14)(5.15)), we first truncate the physical domain of the problem to a rectangle in $2 \mathrm{D}$ or a box in 3D with homogenerous Dirichlet boundary conditions, and then apply backward Euler for time discretization and second-order centered finite difference for spatial derivatives. For more details, we refer to [6] for a similar problem.

\section{Numerical results}

In this section, we will show how to choose initial data for computing ground state of a rotating BEC, and present numerical results of ground, symmetric, central vortex and central vortex ground states, as well as their energy diagrams in 2D and $3 \mathrm{D}$. Furthermore, we also study numerical dimension reduction from 3D to 2D, errors between ground state and its TF approximation and critical angular speed. Due to lemma 3.1, theorem 3.2, we only present results for $0 \leq \Omega<\gamma_{x y}$. In all computations, the bounded computational domain $\Omega_{\mathbf{x}}$ is chosen as a rectangle in $2 \mathrm{D}$ and a box in $3 \mathrm{D}$, which is centered at the origin.

6.1. Initial data for computing ground state. For computing ground state of a rotating BEC, as discussed in section 5, the CNGF and its BEFD discretization guarantees energy diminishing. For $|\Omega| \ll \gamma_{x y}$, i.e. a slowly rotating BEC, the initial data can be chosen as the ground state of (2.8) with $\beta_{d}=0$ and $\Omega=0$ (3.16), which is given explicitly by (3.16). When $\Omega$ increases, the ground state may break symmetry. From our primary numerical study, when $\Omega$ is near $\gamma_{x y}$, the above choice no longer gives the ground state. Here we present a $2 \mathrm{D}$ example to evolve the CNGF (5.11) with its BEFD discretization for four different initial data. 


\begin{tabular}{cccccc}
\hline & $\Omega$ & case I & case II & case III & case IV \\
\hline$\beta_{2}=10$ & 0.0 & 2.3563 & 1.5914 & 1.5914 & 1.5914 \\
& 0.25 & 2.1068 & 1.5914 & 1.5914 & 1.5914 \\
& 0.50 & 1.8572 & 1.5914 & 1.5914 & 1.5914 \\
& 0.75 & 1.6077 & 1.5914 & 1.6078 & 1.5914 \\
& 0.80 & 1.5578 & 1.5914 & 1.5578 & 1.5578 \\
& 0.90 & 1.4580 & 1.4716 & 1.4580 & 1.4580 \\
\hline$\beta_{2}=100$ & 0.00 & 4.3585 & 3.945 & 3.945 & 3.945 \\
& 0.25 & 4.1096 & 3.9456 & 3.945 & 3.945 \\
& 0.50 & 3.8608 & 3.9456 & 3.8608 & 3.8608 \\
& 0.75 & 3.371 & 3.3750 & 3.3802 & 3.371 \\
& 0.80 & 3.2032 & 3.1980 & 3.1817 & 3.1817 \\
& 0.90 & 2.6535 & 2.6573 & 2.6483 & 2.6420 \\
\hline$\beta_{2}=400$ & 0.0 & 7.8995 & 7.6328 & 7.6496 & 7.6328 \\
& 0.25 & 7.6496 & 7.6328 & 7.6328 & 7.6328 \\
& 0.50 & 7.3998 & 7.6328 & 7.2426 & 7.2426 \\
& 0.75 & 5.9993 & 5.9678 & 5.9604 & 5.9603 \\
& 0.80 & 5.5697 & 5.6026 & 5.5481 & 5.5479 \\
& 0.90 & 4.5447 & 4.4651 & 4.4563 & 4.4563 \\
\hline
\end{tabular}

TABLE 6.1. Energy of the steady state solutions of the CNGF (5.11) under the BEFD discretization with four different initial data.

Example 1. For different choices of initial data for CNGF in 2D, i.e. we choose $d=2, \gamma_{x}=\gamma_{y}=1$ and $\beta_{2}=100$ in (5.11). We compute numerically the steady state solution of (5.11) under the BEFD discretization with a bounded computational domain $\Omega_{\mathbf{x}}=[-6,6] \times[-6,6]$ and four different initial data:
I. $\phi_{0}(x, y)=\phi_{\mathrm{ho}}^{v}(x, y)=\frac{x+i y}{\sqrt{\pi}} e^{-\left(x^{2}+y^{2}\right) / 2},(x, y) \in \Omega_{\mathbf{x}}$,
II. $\phi_{0}(x, y)=\phi_{\mathrm{ho}}(x, y)=\frac{1}{\sqrt{\pi}} e^{-\left(x^{2}+y^{2}\right) / 2},(x, y) \in \Omega_{\mathbf{x}}$,
III. $\phi_{0}(x, y)=\frac{\left[\phi_{\mathrm{ho}}(x, y)+\phi_{\mathrm{ho}}^{v}(x, y)\right] / 2}{\left\|\left[\phi_{\mathrm{ho}}(x, y)+\phi_{\mathrm{ho}}^{v}(x, y)\right] / 2\right\|},(x, y) \in \Omega_{\mathbf{x}}$,
IV. $\phi_{0}(x, y)=\frac{(1-\Omega) \phi_{\mathrm{ho}}(x, y)+\Omega \phi_{\mathrm{ho}}^{v}(x, y)}{\left\|(1-\Omega) \phi_{\mathrm{ho}}(x, y)+\Omega \phi_{\mathrm{ho}}^{v}(x, y)\right\|},(x, y) \in \Omega_{\mathbf{x}}$.

The steady state solution is obtained numerically when $\max _{j, l}\left|\phi_{j, l}^{n+1}-\phi_{j, l}^{n}\right|<\epsilon=$ $10^{-7}$. Table 6.1 shows the energy of the steady state solutions with four different initial data for $0 \leq \Omega<\gamma_{x y}=1$ and $\beta_{2}=10,100$ and 400 .

From Table 6.1 and our additional numerical experiments, we have the following observations: (i) Type IV initial data gives the ground state for all $|\Omega|<\gamma_{x y}=1$, (ii) Types II\& III initial data only give the ground state when $|\Omega|$ is near 0 , (iii) Type I initial data only gives the ground state when $|\Omega|$ is less than but very near $\gamma_{x y}=1$.

From this numerical study for computing ground state, when $\gamma_{x}=\gamma_{y}=1$ and $\gamma_{z}=O(1)$, we suggest choosing the following initial data in (5.12) for the CNGF (5.11) with its BEFD discretization:

$$
\phi_{0}(\mathbf{x})=\frac{(1-\Omega) \phi_{\mathrm{ho}}(\mathbf{x})+\Omega \phi_{\mathrm{ho}}^{v}(\mathbf{x})}{\left\|(1-\Omega) \phi_{\mathrm{ho}}(\mathbf{x})+\Omega \phi_{\mathrm{ho}}^{v}(\mathbf{x})\right\|}, \quad \mathbf{x} \in \mathbb{R}^{d}
$$


where

$$
\phi_{\mathrm{ho}}(\mathbf{x})=\left\{\begin{array}{l}
\frac{1}{\sqrt{\pi}} e^{-\frac{x^{2}+y^{2}}{2}} \\
\frac{\gamma_{z}^{1 / 4}}{\pi^{\frac{3}{4}}} e^{-\frac{x^{2}+y^{2}+\gamma_{z} z^{2}}{2}}
\end{array} \phi_{\mathrm{ho}}^{v}(\mathbf{x})=\left\{\begin{array}{l}
\frac{1}{\sqrt{\pi}}(x+i y) e^{-\frac{x^{2}+y^{2}}{2}} \quad d=2, \\
\frac{\gamma_{z}^{1 / 4}}{\pi^{\frac{3}{4}}}(x+i y) e^{-\frac{x^{2}+y^{2}+\gamma_{z} z^{2}}{2}} d=3 .
\end{array}\right.\right.
$$

Under this choice of initial data, the continuation technique is no longer required for computing the ground state. Furthermore, from our numerical study, the initial data for computing the central vortex ground state can be chosen as

$$
\phi_{0}(\mathbf{x})=\phi_{\mathrm{ho}}^{v}(\mathbf{x}), \quad \mathbf{x} \in \mathbb{R}^{d} .
$$

For how to choose initial data in 3D for a prolate trap in order to obtain a bending vortex, we refer to $[3,21,32,33,35,44]$.

\subsection{Results in $2 \mathrm{D}$.}

Example 2. For ground, symmetric and central vortex states, as well as their energy configurations, in 2D, i.e. we take $d=2$ and $\gamma_{x}=\gamma_{y}=1$ in (2.8). Figure 6.1 plots surface and contour of the ground state $\phi^{g}(x, y):=\phi_{\beta, \Omega}^{g}(x, y)$ with $\beta_{2}=100$ for different $\Omega$ and Figure 6.2 for the central vortex ground state $\phi^{v}(x, y):=\phi_{\beta, \Omega}^{v}(x, y)$. Figure 6.3 plots the symmetric state $\phi^{0}(r):=\phi_{\beta, 0}^{0}(r)$ and first three central vortex states $\phi^{m}(r):=\phi_{\beta, 0}^{m}(r)(m=1,2,3)$ for different interaction rate $\beta_{2}$. Table 6.2 and Figure 6.4 show the energy configurations of these states for different $\beta_{2}$ and $\Omega$. Furthermore, Figure 6.5 shows ratios between the energy of these states, and Figure 6.6 plots the energy of the ground state when $\beta_{2} \gg 1$ and $\Omega \approx \gamma_{x y}=1$. Similar study for the ground state and its energy diagram has been done in the physical literatures [1].

From Figs. 6.1-6 and Table 6.2, we reach the following conjectures for a rotating BEC under an isotropic trap in 2 D, i.e. $\gamma_{x}=\gamma_{y}=1$ :

(a) For any fixed $\beta_{2}>0$, there exists a critical angular frequency $0<\Omega^{c}<\gamma_{x y}=1$ such that: (i) when $0 \leq \Omega<\Omega^{c}$, the ground state $\phi_{\beta, \Omega}^{g}=\phi_{\beta, 0}^{g}=\phi_{\beta, 0}^{0}, E_{\beta, \Omega}^{g}=E_{\beta, 0}^{g}=E_{\beta, 0}^{0}$ and $\mu_{\beta, \Omega}^{g}=\mu_{\beta, 0}^{g}=\mu_{\beta, 0}^{0}$; (ii) when $\Omega=\Omega^{c}$, the ground state is not unique, e.g. both $\phi_{\beta, 0}^{0}$ and $\phi_{\beta, 0}^{1}$ are ground states, i.e. $E_{\beta, \Omega^{c}}^{g}=E_{\beta, 0}^{0}=E_{\beta, \Omega^{c}}^{1}$ and $\mu_{\beta, \Omega^{c}}^{g}=\mu_{\beta, 0}^{0}=\mu_{\beta, \Omega^{c}}^{1}$; (iii) when $\Omega^{c}<\Omega<\gamma_{x y}=1$, quantized vortices appear in the ground states, and the larger $\Omega$ is the more vortices appear in the ground state. A similar conclusion was observed in the literatures $[1,40,41]$.

(b) Similar patterns for the central vortex ground state are observed as in (a) for the ground state.

(c) For any fixed $\beta_{2} \geq 0$, when $\Omega$ increases from 0 to $\gamma_{x y}=1$, the energy $E_{\beta, \Omega}^{s}$ and chemical potential $\mu_{\beta, \Omega}^{s}(s=g, v, 0,1, \cdots)$ of any stationary states decrease. Furthermore, the energy of the ground state has the following asymptotics when $\Omega$ is near $\gamma_{x y}=1$ (cf. Fig. 6.6b):

$$
E_{\beta, \Omega}^{g}=E_{\beta, \pm \gamma_{x y}^{\mp}}^{g}+O\left(\gamma_{x y} \mp \Omega\right), \quad \Omega \rightarrow \pm \gamma_{x y}^{\mp} .
$$

(d) For any fixed $|\Omega|<\gamma_{x y}=1$, when $\beta_{2}$ increases, the energy $E_{\beta, \Omega}^{s}$ and chemical potential $\mu_{\beta, \Omega}^{s}(s=g, v, 0,1, \cdots)$ of any stationary states increase too, and satisfy

$$
\begin{aligned}
& \lim _{\beta_{2} \rightarrow \infty} \frac{E_{\beta, \Omega}^{v}}{E_{\beta, \Omega}^{g}}=\lim _{\beta_{2} \rightarrow \infty} \frac{\mu_{\beta, \Omega}^{v}}{\mu_{\beta, \Omega}^{g}}=1, \lim _{\beta_{2} \rightarrow \infty} \frac{E_{\beta, \Omega}^{s}}{E_{\beta, \Omega}^{g}}=\text { const, } \lim _{\beta_{2} \rightarrow \infty} \frac{\mu_{\beta, \Omega}^{s}}{\mu_{\beta, \Omega}^{g}}=\text { const, } s \geq 0, \\
& E_{\beta, \Omega}^{s}=O\left(\beta_{2}^{1 / 2}\right), \mu_{\beta, \Omega}^{s}=O\left(\beta_{2}^{1 / 2}\right) \text {, when } \beta_{2} \rightarrow \infty, s=g, v, 0,1, \cdots .
\end{aligned}
$$


$\Omega=0.0$

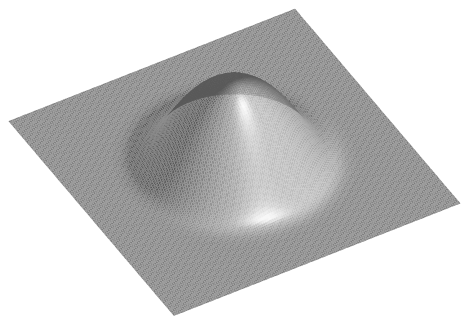

$\Omega=0.50$

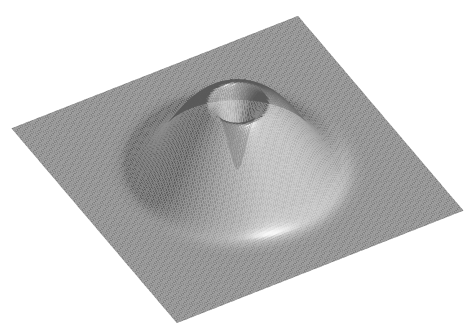

$\Omega=0.80$

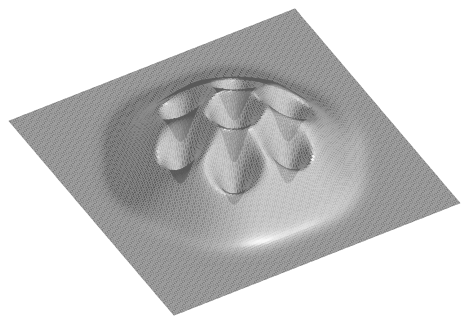

$\Omega=0.25$

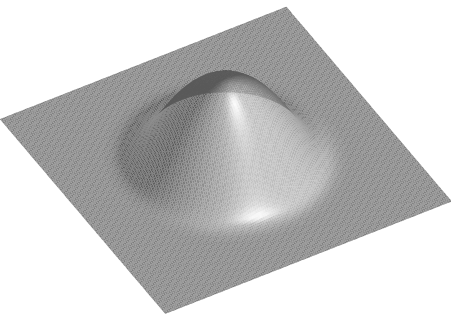

$\Omega=0.70$

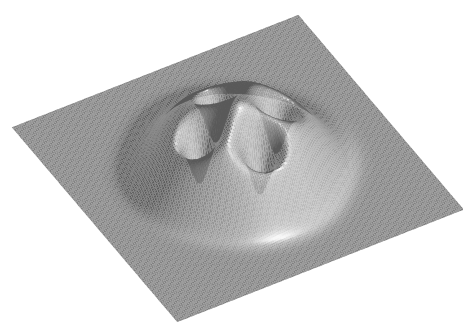

$\Omega=0.95$

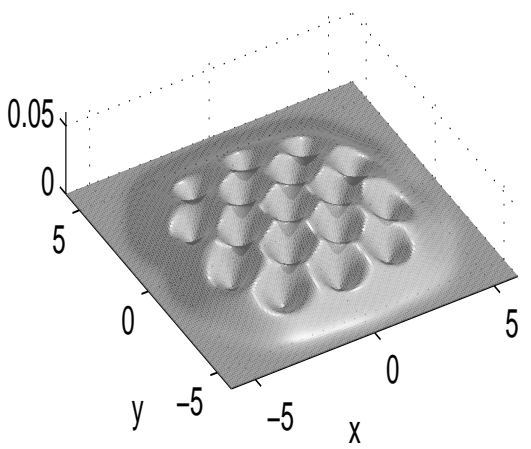

FIG. 6.1. Surface plots of ground state density function $\left|\phi^{g}(x, y)\right|^{2}$ in $2 D$ with $\gamma_{x}=\gamma_{y}=1$ and $\beta_{2}=100$ for different $\Omega$ in Example 2.

Example 3. For ground state in 2D with nonsymmetric trap, i.e., we take $d=2$, $\gamma_{x}=1$ and $\gamma_{y}=1.5$ in (2.8). Figure 6.7 plots the surface of the ground state $\phi^{g}(x, y)$ with $\beta_{2}=100$ for different $\Omega$. We notice this case was also studied in [1] by using a different numerical method.

From Fig. 6.7, the conjecture (a) for ground states in Example 2 is still valid for an anisotropic trap, i.e. $1=\gamma_{x} \neq \gamma_{y}>1$, in 2 D. 

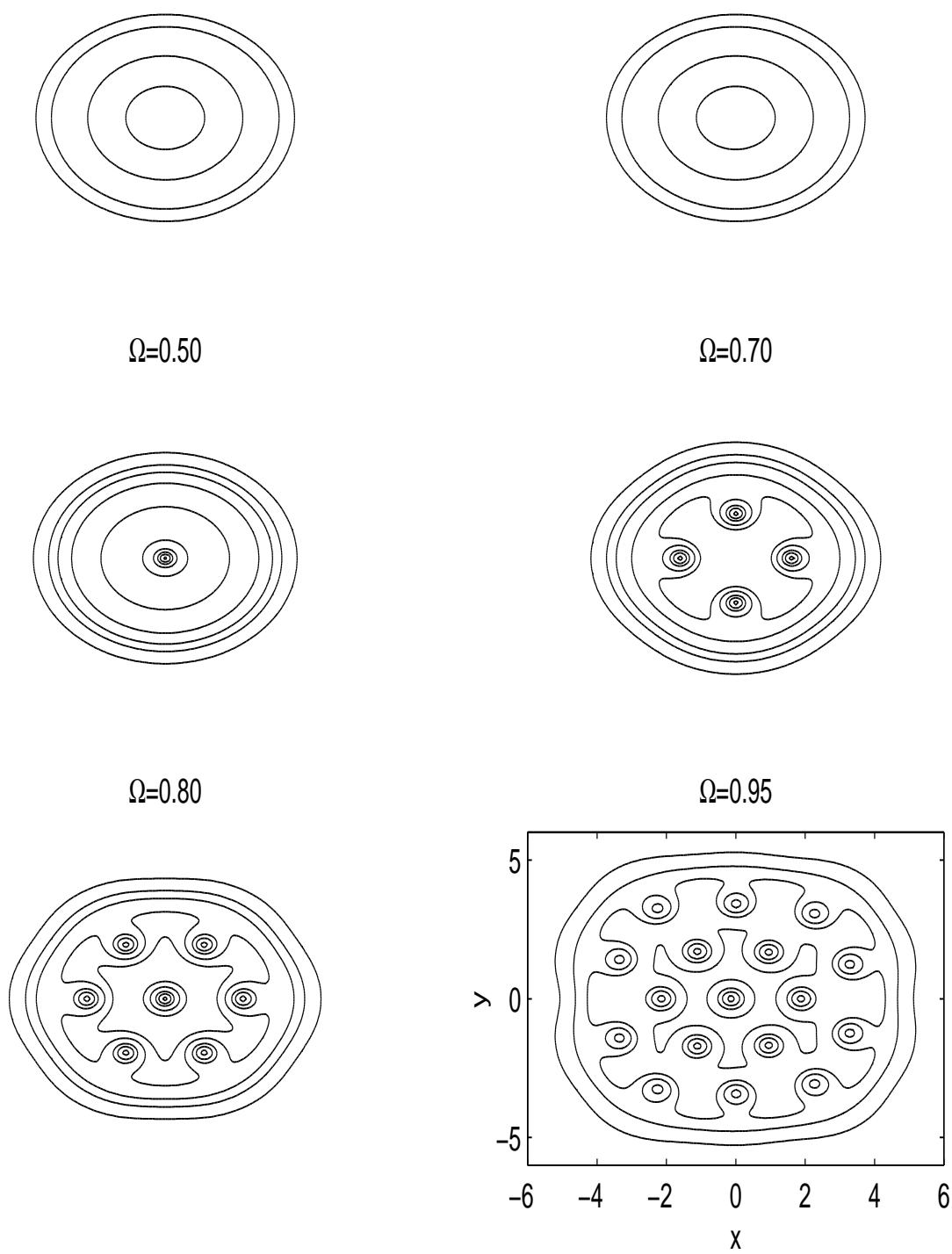

Figure 6.1(cont'd): Contour plots.

\subsection{Results in $3 \mathrm{D}$.}

Example 4. For ground, symmetric and central vortex states, as well as their energy configurations, in 3D, i.e. we take $d=3, \gamma_{x}=1, \gamma_{y}=1$ and $\gamma_{z}=1$ in (2.8). Figure 6.8 plots the isosurface of the ground state $\phi^{g}(x, y, z):=\phi_{\beta, \Omega}^{g}(x, y, z)$ and central vortex ground state $\phi^{v}(x, y, z):=\phi_{\beta, \Omega}^{v}(x, y, z)$ for different $\Omega$. Figure 6.9 plots the symmetric state $\phi^{0}(r, z=0), \phi^{0}(r=0, z)$, and the first central vortex state $\phi^{1}(r, z=0)$, $\phi^{1}(r=0, z)$ for different interaction rate $\beta$. Figure 6.10 shows the energy configurations of these states for different $\beta$ and $\Omega$ in $3 \mathrm{D}$. 
$\Omega=0.0$

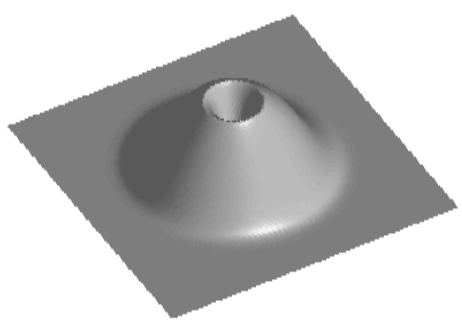

$\Omega=0.50$

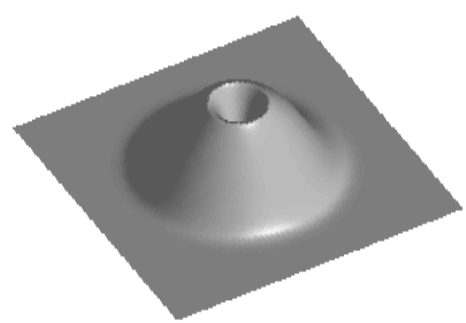

$\Omega=0.80$

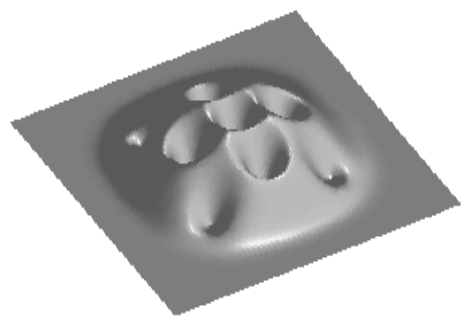

$\Omega=0.25$

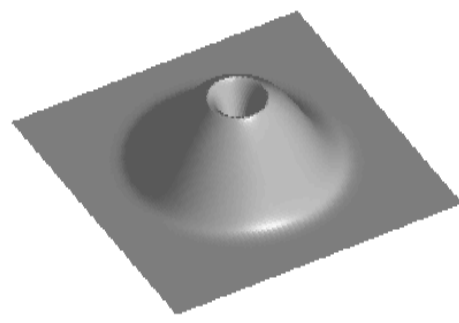

$\Omega=0.70$

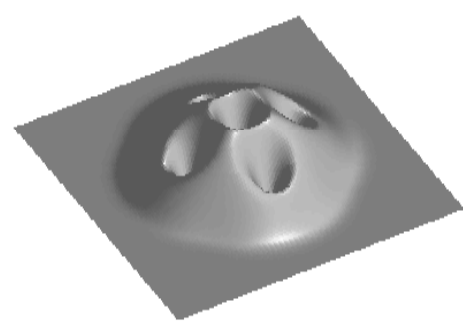

$\Omega=0.90$

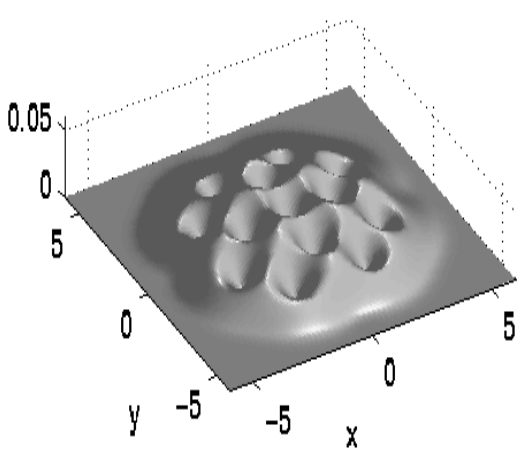

FIG. 6.2. Surface plots of central vortex ground state density function $\left|\phi^{v}(x, y)\right|^{2}$ in $2 D$ with $\gamma_{x}=\gamma_{y}=1$ and $\beta_{2}=100$ for different $\Omega$ in Example 2 .

From Figs. 6.8-10, all the conjectures at Example 2 are still valid in 3D except that (6.3) should be modified to

$$
E_{\beta, \Omega}^{s}=O\left(\beta^{2 / 5}\right), \mu_{\beta, \Omega}^{s}=O\left(\beta^{2 / 5}\right), \text { when } \beta \rightarrow \infty, \quad s=g, v, 0,1, \cdots .
$$

For obtaining a bending vortex, e.g. S-shaped vortex and U-shaped vortex, in 3D with a prolate trap, we refer to $[3,21,32,33,35,44]$. 
a)

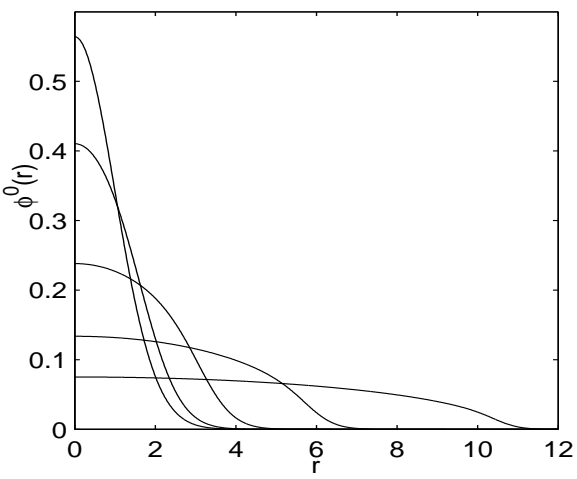

c)

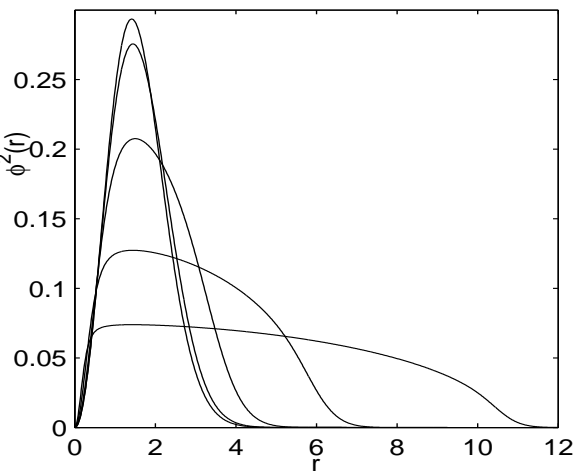

b)

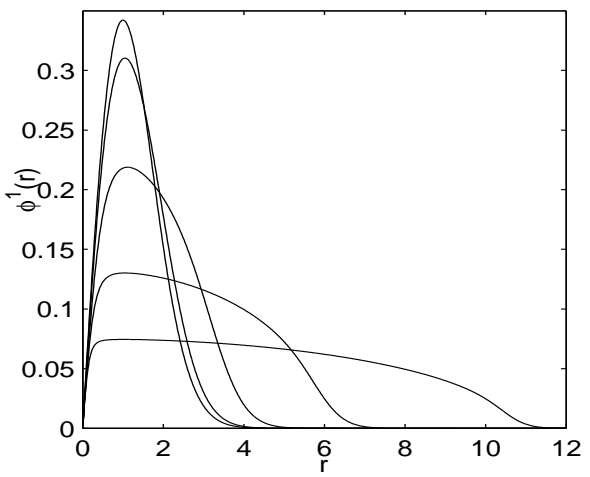

d)

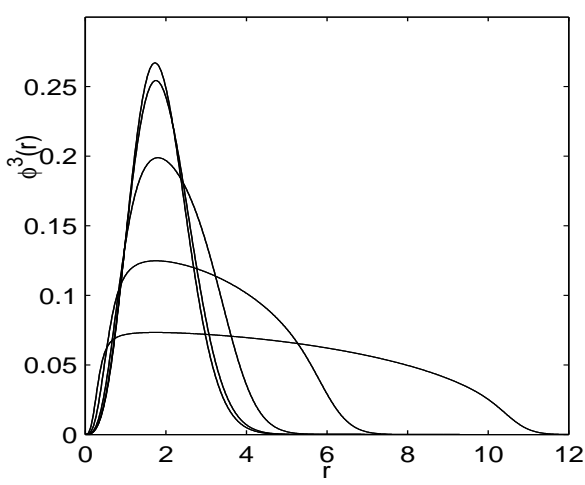

FIG. 6.3. Symmetric and central vortex states in $2 D$ with $\gamma_{x}=\gamma_{y}=1$ for $\beta_{2}=0,10,100,1000$, 10000 (in the order of decreasing of peak) in Example 2. Symmetric state $\phi^{0}(r)$ : a); and central vortex states $\left.\left.\phi^{m}(r): b\right) . m=1, c\right) . m=2$ and $\left.d\right) . m=3$.

\subsection{Critical angular velocity.}

Example 5. For critical angular velocities in 2D, i.e. we take $d=2$ and $\gamma_{x}=$ $\gamma_{y}=1$ in (2.8). Table 6.3 displays the critical angular velocity $\Omega^{c}:=\Omega_{\beta}^{c}$ and $\Omega^{v}:=\Omega_{\beta}^{v}$ for different $\beta_{2}$.

From Table 6.3, we get: (a) $\Omega^{c}=\Omega^{v}=\gamma_{x y}=1.0$ when $\beta_{2}=0$ and $0<\Omega^{c}<\Omega^{v}<$ $\gamma_{x y}=1$ when $\beta_{2}>0$; (b) $\Omega^{c}$ and $\Omega^{v}$ decrease when $\beta_{2}$ increases; (c) $\Omega^{c} \approx E_{\beta, 0}^{1}-E_{\beta, 0}^{0}$ and $\Omega^{v} \approx E_{\beta, 0}^{2}-E_{\beta, 0}^{1}$ for $\beta_{2} \geq 0$. All these results agree very well with the numerical results $[23,26]$ and analytical estimate $[40,41]$ in the literature. Similar observations are still valid in 3D.

6.5. Numerical verification of dimension reduction. As we know, when $\gamma_{x}=O(1), \gamma_{y}=O(1)$ and $\gamma_{z} \gg 1$, the 3D GPE can be asymptotically reduced to a $2 \mathrm{D}$ GPE. Here we present a numerical verification of this procedure. In order to do so, we compute the ground state $\phi_{g}^{3 \mathrm{D}}(x, y, z)$ of the $3 \mathrm{D}$ GPE, i.e. we take $d=3, \gamma_{x}=\gamma_{y}=1$ 


\begin{tabular}{cccccccc}
\hline & $\beta$ & $E_{\beta, \Omega}^{g}$ & $E_{\beta, \Omega}^{0}$ & $E_{\beta, \Omega}^{v}$ & $E_{\beta, \Omega}^{1}$ & $E_{\beta, \Omega}^{2}$ & $E_{\beta, \Omega}^{3}$ \\
\hline$\Omega=0.0$ & 0 & 0.997 & 0.999 & 1.993 & 1.999 & 2.999 & 3.999 \\
& 10.0 & 1.591 & 1.592 & 2.356 & 2.361 & 3.280 & 4.237 \\
& 100.0 & 3.945 & 3.945 & 4.358 & 4.368 & 5.040 & 5.821 \\
& 1000.0 & 11.970 & 11.971 & 12.151 & 12.166 & 12.544 & 13.039 \\
\hline$\Omega=0.50$ & 0.0 & 0.997 & 0.999 & 1.494 & 1.499 & 1.999 & 2.499 \\
& 10.0 & 1.591 & 1.592 & 1.857 & 1.861 & 2.280 & 2.737 \\
& 100.0 & 3.860 & 3.945 & 3.860 & 3.868 & 4.040 & 4.321 \\
& 1000.0 & 11.02 & 11.971 & 11.02 & 11.366 & 11.544 & 11.539 \\
\hline$\Omega=0.75$ & 0 & 0.997 & 0.999 & 1.245 & 1.249 & 1.499 & 1.749 \\
& 10.0 & 1.591 & 1.592 & 1.608 & 1.611 & 1.780 & 1.987 \\
& 100.0 & 3.371 & 3.945 & 3.371 & 3.618 & 3.540 & 3.571 \\
& 1000.0 & 9.098 & 11.971 & 9.114 & 11.416 & 11.044 & 10.789 \\
\hline$\Omega=0.90$ & 0 & 0.991 & 0.999 & 1.095 & 1.099 & 1.199 & 1.299 \\
& 10.0 & 1.449 & 1.592 & 1.458 & 1.461 & 1.480 & 1.537 \\
& 100.0 & 2.648 & 3.945 & 2.653 & 3.468 & 3.240 & 3.121 \\
& 1000.0 & 7.163 & 11.971 & 7.176 & 11.266 & 10.744 & 10.339 \\
\hline \multirow{5}{*}{$\Omega(1649$}
\end{tabular}

TABLE 6.2. Energy of the ground state $E_{\beta, \Omega}^{g}$, symmetric state $E_{\beta, \Omega}^{0}$, central vortex ground state $E_{\beta, \Omega}^{v}$ and the first three central vortex states $E_{\beta, \Omega}^{j}(j=1,2,3)$ with $\gamma_{x}=\gamma_{y}=1$ for different $\beta_{2}$ and $\Omega$ in $2 D$ in Example 2.

\begin{tabular}{ccccc}
\hline$\beta_{2}$ & 0 & 10 & 100 & 1000 \\
$\Omega^{c}\left(\approx E_{\beta, 0}^{1}-E_{\beta, 0}^{0}\right)$ & $1.0(1.0)$ & $0.775(0.769)$ & $0.406(0.423)$ & $0.188(0.195)$ \\
$\Omega^{v}\left(\approx E_{\beta, 0}^{2}-E_{\beta, 0}^{1}\right)$ & $1.0(1.0)$ & $0.87(0.924)$ & $0.625(0.682)$ & $0.375(0.393)$ \\
\hline
\end{tabular}

TABLE 6.3. Critical angular velocities in $2 D$.

and $\beta=100$ in (2.8), numerically for different $\gamma_{z}$. Then we compute

$$
\begin{aligned}
& \phi_{3}(z)=\sqrt{\int_{\mathbb{R}^{2}}\left|\phi_{g}^{3 \mathrm{D}}(x, y, z)\right|^{2} d x d y} \approx \phi_{\mathrm{ho}}(z)=\frac{\gamma_{z}^{1 / 4}}{\pi^{1 / 4}} e^{-\gamma_{z} z^{2} / 2}, \\
& \beta_{2}=\beta \int_{-\infty}^{\infty}\left|\phi_{3}(z)\right|^{4} d z \approx \beta_{2}^{a}=\beta \sqrt{\gamma_{z} / 2 \pi} .
\end{aligned}
$$

We also find the ground state $\phi_{g}^{2 \mathrm{D}}(x, y)$ of the 2D GPE, i.e. we take $d=2, \gamma_{x}=\gamma_{y}=1$ and $\beta_{2}=100 \sqrt{\gamma_{z} / 2 \pi}$ in (2.8), numerically for different $\gamma_{z}$. Table 6.4 lists the errors of $\beta_{2}-\beta_{2}^{a}, \max \left|\phi_{3}-\phi_{\mathrm{ho}}\right|,\left\|\phi_{3}-\phi_{\mathrm{ho}}\right\|_{L^{2}}, \max \left|\phi_{g}^{3 \mathrm{D}}-\phi_{g}^{2 \mathrm{D}} \phi_{\mathrm{ho}}\right|$ and $\left\|\phi_{g}^{3 \mathrm{D}}-\phi_{g}^{2 \mathrm{D}} \phi_{\mathrm{ho}}\right\|_{L^{2}}$ for different $\gamma_{z}$.

From Table 6.4, the dimension reduction of GPE from 3D to $2 \mathrm{D}$ when $\gamma_{z} \gg 1$ is verified numerically. Furthermore, for fixed $\beta>0$, when $\gamma_{z} \rightarrow \infty$, we have the following 
a)

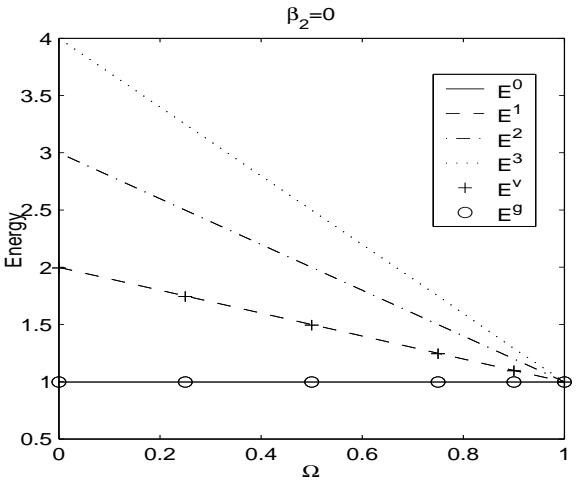

c)

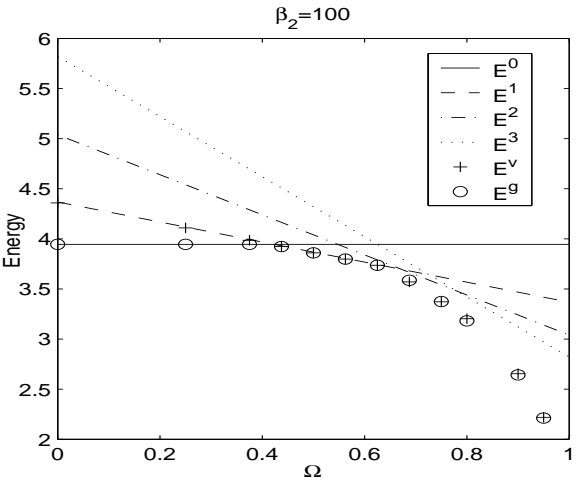

b)
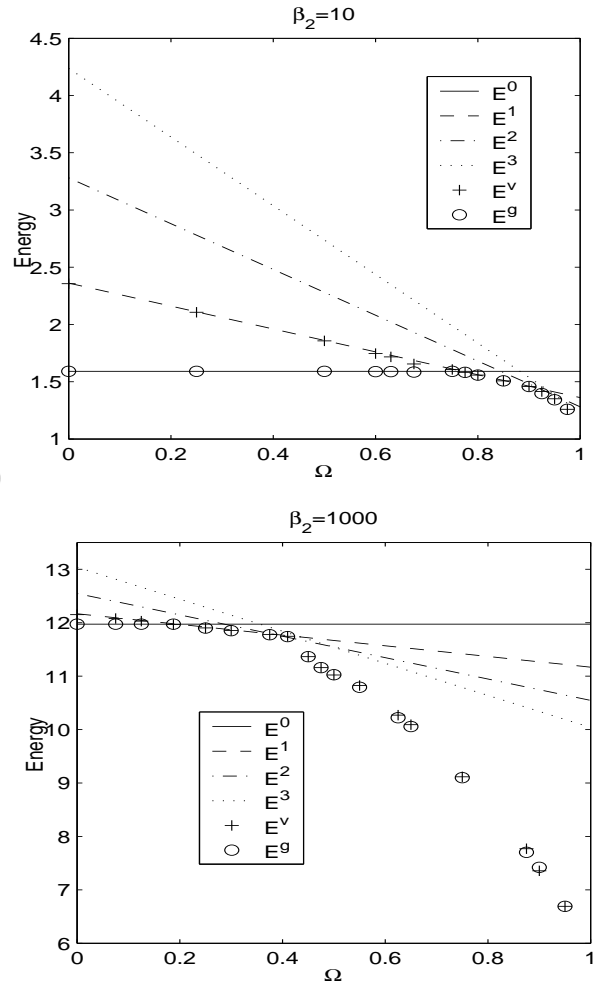

FIG. 6.4. Energy diagrams for ground state, i.e. $E^{g}:=E_{\beta, \Omega}^{g}$, symmetric state, i.e. $E^{0}:=E_{\beta, \Omega}^{0}$, central vortex states, i.e. $E^{m}:=E_{\beta, \Omega}^{m}$, and central vortex ground state, i.e. $E^{v}:=E_{\beta, \Omega}^{v}$, in $2 D$ with $\gamma_{x}=\gamma_{y}=1$ for different $\beta_{2}$ and $\Omega$ in Example 2 .

\begin{tabular}{lccc}
\hline $1 / \gamma_{z}$ & $1 / 4$ & $1 / 8$ & $1 / 16$ \\
\hline$\frac{\left|\beta_{2}-\beta_{2}^{a}\right|}{\beta_{2}}$ & 0.1188 & 0.0822 & 0.0524 \\
$\left\|\phi_{3}-\phi_{\text {ho }}\right\|_{L^{\infty}}$ & 0.084 & 0.066 & 0.048 \\
$\left\|\phi_{3}-\phi_{\text {ho }}\right\|_{L^{2}}$ & 0.097 & 0.062 & 0.038 \\
$\left\|\phi_{g}^{3 \mathrm{D}}-\phi_{g}^{2 \mathrm{D}} \phi_{\mathrm{ho}}\right\|_{L^{\infty}}$ & 0.083 & 0.025 & 0.015 \\
$\left\|\phi_{g}^{3 \mathrm{D}}-\phi_{g}^{2 \mathrm{D}} \phi_{\mathrm{ho}}\right\|_{L^{2}}$ & 0.260 & 0.074 & 0.047 \\
\hline
\end{tabular}

TABLE 6.4. Numerical verification of dimension reduction from $3 D$ to $2 D$.

convergence rate:

$$
\begin{aligned}
& \frac{\beta_{2}-\beta_{2}^{a}}{\beta_{2}}=O\left(\frac{\ln \gamma_{z}}{\gamma_{z}^{3 / 4}}\right), \quad \beta_{2}=\beta \sqrt{\frac{\gamma_{z}}{2 \pi}}\left(1+O\left(\frac{\ln \gamma_{z}}{\gamma_{z}^{3 / 4}}\right)\right), \\
& \left\|\phi_{3}-\phi_{\mathrm{ho}}\right\|_{L^{\infty}}=O\left(\frac{\ln \gamma_{z}}{\gamma_{z}^{1 / 2}}\right), \quad\left\|\phi_{3}-\phi_{\mathrm{ho}}\right\|_{L^{2}}=O\left(\frac{\ln \gamma_{z}}{\gamma_{z}^{3 / 4}}\right), \\
& \left\|\phi_{g}^{3 \mathrm{D}}-\phi_{g}^{2 \mathrm{D}} \phi_{\mathrm{ho}}\right\|_{L^{\infty}}=O\left(\frac{\ln \gamma_{z}}{\gamma_{z}^{5 / 8}}\right), \quad\left\|\phi_{g}^{3 \mathrm{D}}-\phi_{g}^{2 \mathrm{D}} \phi_{\mathrm{ho}}\right\|_{L^{2}}=O\left(\frac{\ln \gamma_{z}}{\gamma_{z}^{3 / 4}}\right) .
\end{aligned}
$$



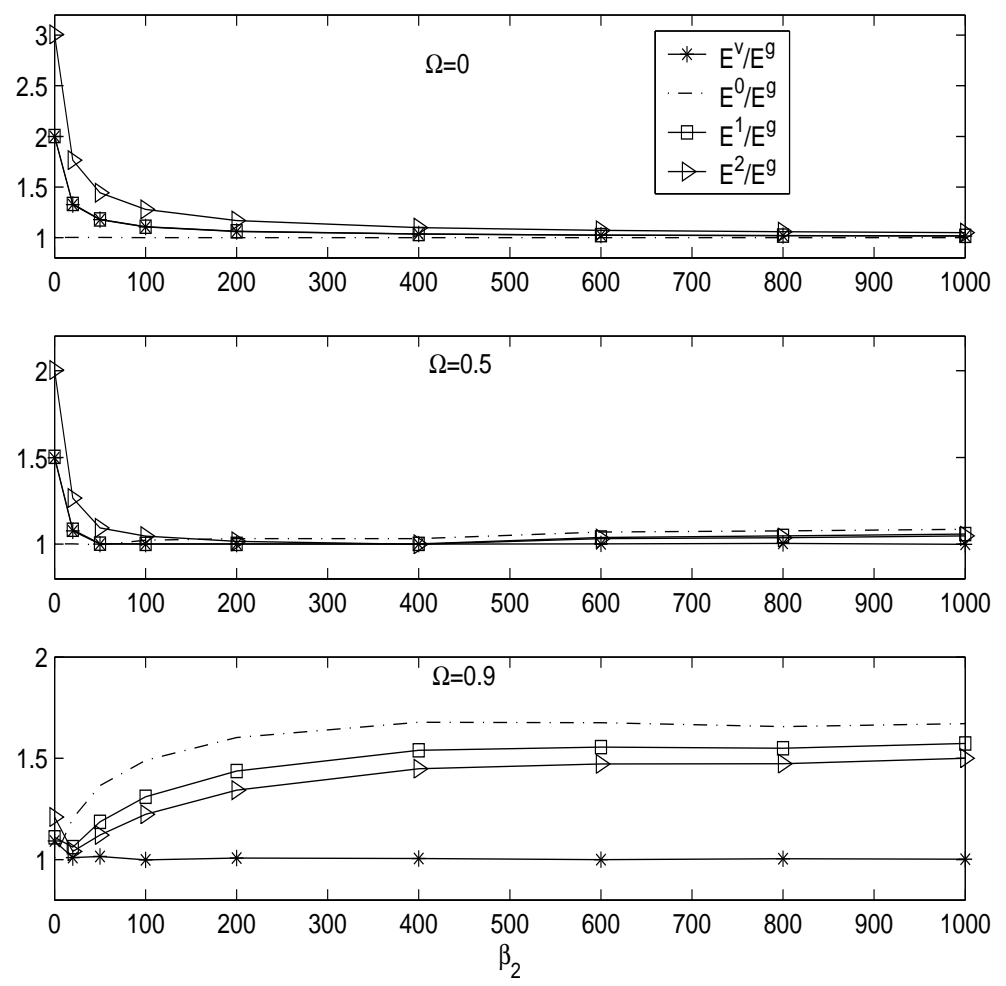

FIG. 6.5. Ratios between the energy of different states in Example 2.

a)

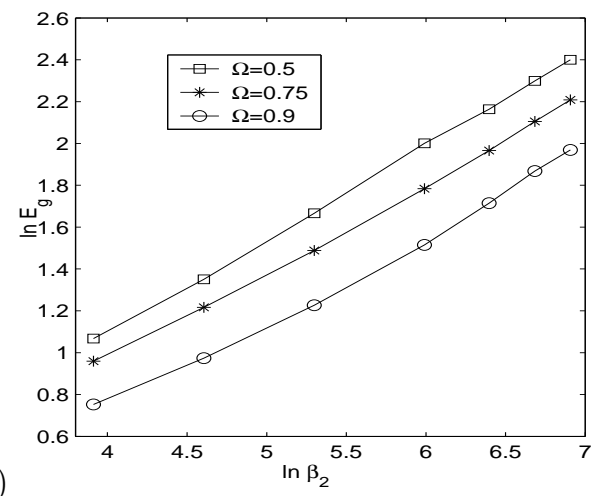

b)

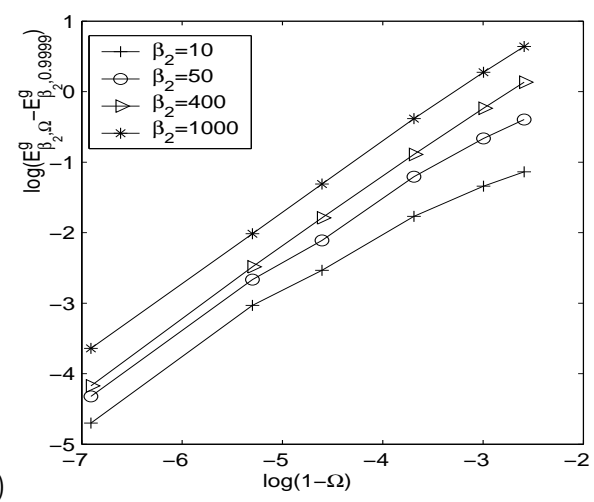

FIG. 6.6. Asymptotics of the energy for the ground state in Example 2. a). When $\beta_{2} \gg 1, b$ ). When $\Omega \approx \gamma_{x y}=1$.

6.6. Errors of the TF approximation. As we know, when $\beta_{d} \gg 1, \gamma_{x}=$ $O(1), \gamma_{y}=O(1), \gamma_{z}=O(1)$ and $|\Omega| \approx 0$, the ground state is very well approximated by the $\mathrm{TF}$ approximation $[1,2,8,9]$. Here we study numerically how effective the TF approximation is, when $|\Omega|$ is large, especially when $|\Omega|$ is near $\gamma_{x y}$. In order to do so, we compare the ground state and its TF approximation in $2 \mathrm{D}$, i.e. we take $d=2$ 
$\Omega=0$

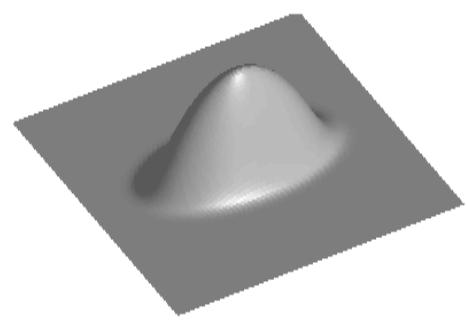

$\Omega=0.50$

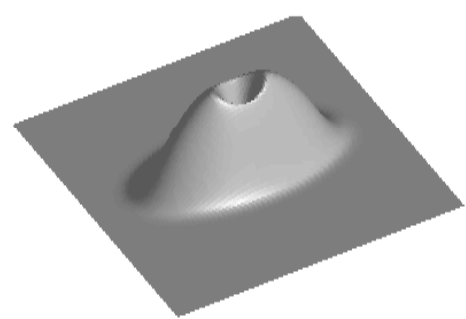

$\Omega=0.90$

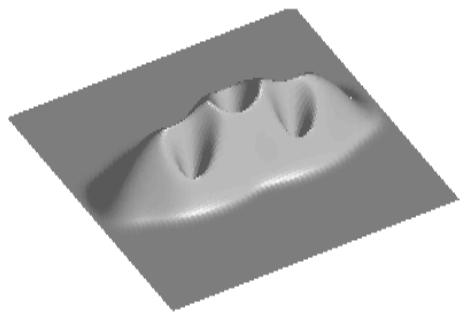

$\Omega=0.25$

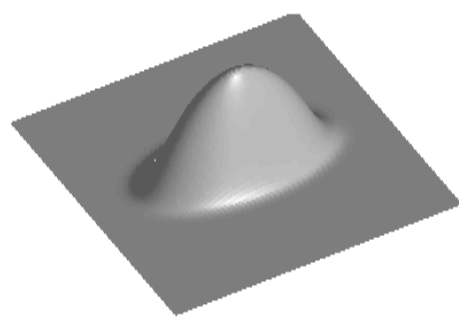

$\Omega=0.75$

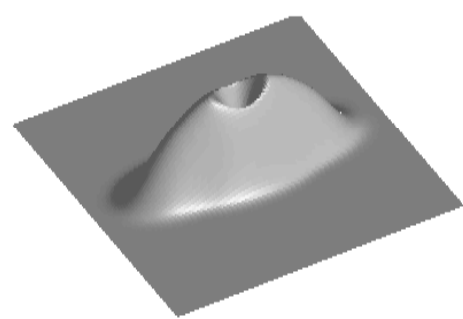

$\Omega=0.999$

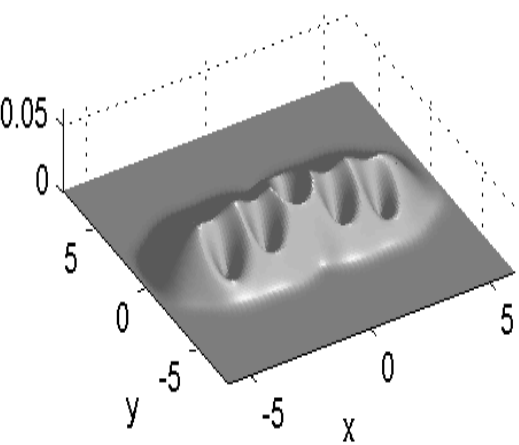

FIG. 6.7. Surface plots of ground state density function $\left|\phi^{g}(x, y)\right|^{2}$ in $2 D$ with $\beta_{2}=100$ in a nonsymmetric trap, i.e. $\gamma_{x}=1$ and $\gamma_{y}=1.5$ for different $\Omega$ in Example 3.

and $\gamma_{x}=\gamma_{y}=1$ in (2.8). Table 6.5 lists the errors $\left.\max || \phi_{\beta, \Omega}^{g}\right|^{2}-\left|\phi_{\beta}^{\mathrm{TF}}\right|^{2} \mid, E_{\beta, \Omega}^{g}-E_{\beta, \Omega}^{\mathrm{TF}}$ and $\mu_{\beta, \Omega}^{g}-\mu_{\beta, \Omega}^{\mathrm{TF}}$ for different $\beta_{2} \gg 1$ and $\Omega$.

From Table 6.5, we can see that the density of the ground state converges to the TF approximation for any $|\Omega|<\gamma_{x y}=1$ when $\beta_{2} \rightarrow \infty$. However, when $\beta_{2}$ increases, the energy and chemical potential of the ground state converge to those of the TF approximation only when $|\Omega| \approx 0$, but diverge when $|\Omega|$ is near $\gamma_{x y}=1$. This is due to 
a)

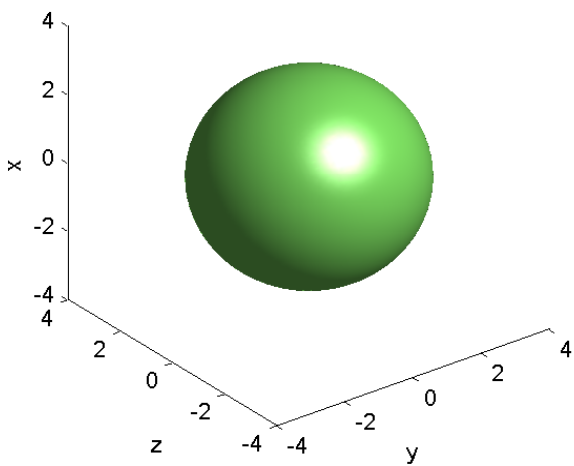

c)

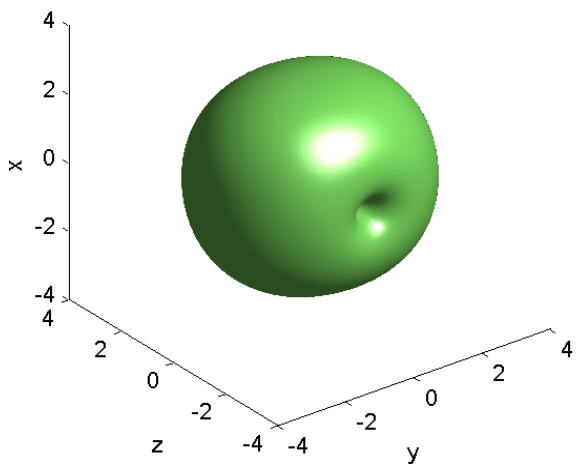

b)

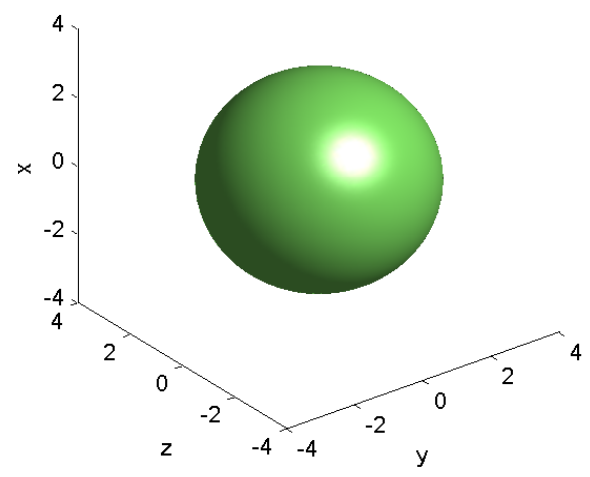

d)

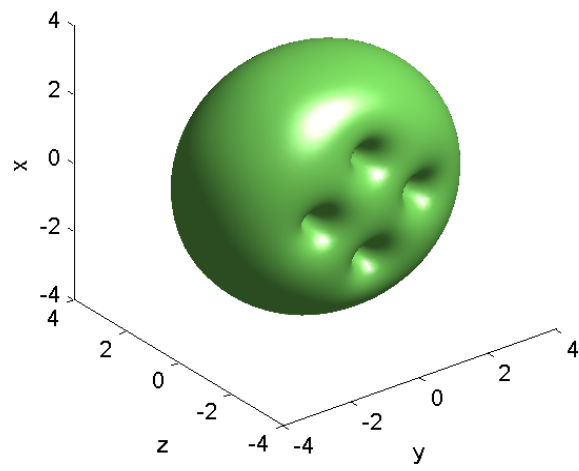

FIG. 6.8. Ground states and central vortex ground states in $3 D$ with $\gamma_{x}=\gamma_{y}=\gamma_{z}=1$ and $\beta_{3}=100$ for different $\Omega$ in Example 4: (a) $\Omega=0$, (b) $\Omega=0.5$, (c) $\Omega=0.8$ and (d) $\Omega=0.9$. Isosurface plots of ground states $\left|\phi^{g}(x, y, z)\right|^{2}=0.001$.

the fact that more and more vortices appear in the ground state when $|\Omega|$ is near 1 and $\beta_{2} \gg 1$. A similar conclusion is also valid in $3 \mathrm{D}$ based on our additional numerical experiments.

6.7. Spurious numerical ground states when $|\Omega|>\gamma_{x y}$. As discussed in section 3, for a rotating BEC, there exists a ground state when $|\Omega|<\gamma_{x y}$ and no ground state when $|\Omega|>\gamma^{x y}$. But when we evolve the discretization of (5.1)-(5.3) with the initial data (6.1) numerically, for any given $\beta_{2} \geq 0$ and $\Omega \in \mathbb{R}$, we always obtain a steady state $\phi_{\beta, \Omega}^{h}$ for a fixed mesh size $h \ll 1$. In fact, this steady state is the minimizer of the following finite-dimensional minimization problem:

$$
E^{h}:=\min _{\phi^{h} \in S^{h}} E_{\beta, \Omega}^{h}\left(\phi^{h}\right),
$$


a)

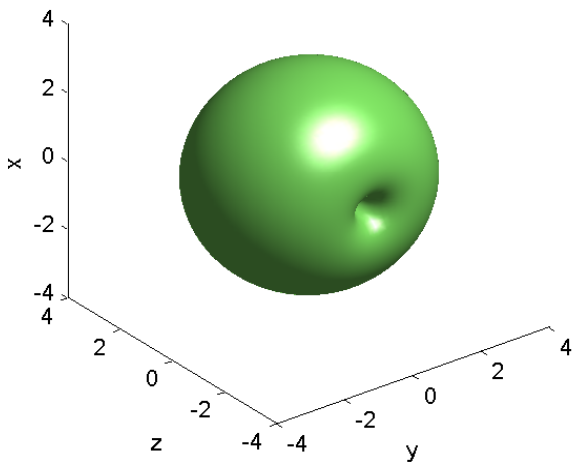

c)

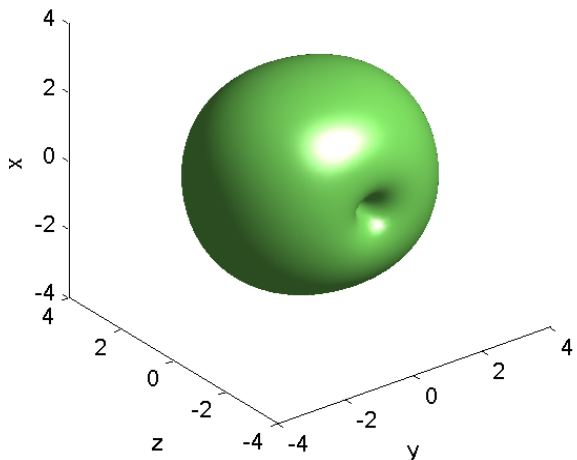

b)

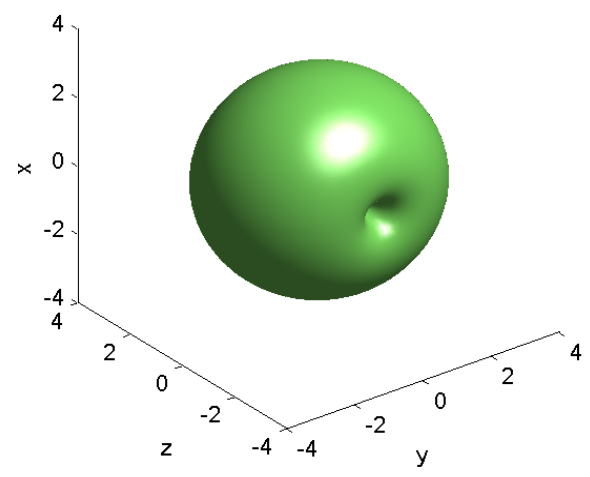

d)

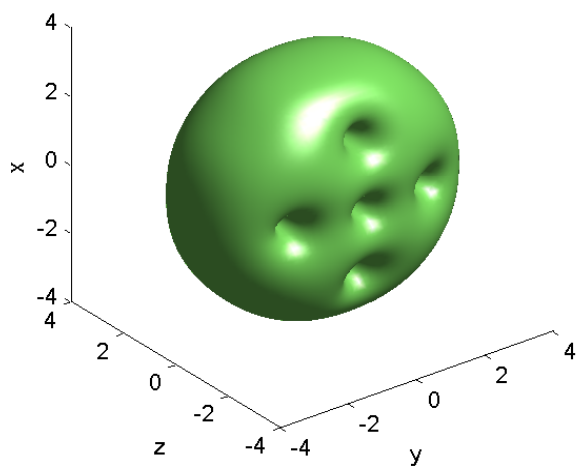

Figure 6.8(cont'd): (iv) Isosurface plots of central vortex ground states $\left|\phi^{v}(x, y, z)\right|^{2}=0.001$.

where $\quad S^{h}=\left\{\phi^{h} \in \mathbb{C}^{(M+1) \times(N+1)} \quad \mid \quad\left\|\phi^{h}\right\|=1, \phi_{0, l}^{h}=\phi_{M, l}^{h}=\phi_{j, 0}^{h}=\phi_{j, N}^{h}=0, \quad j=\right.$ $0, \cdots, M, l=0, \cdots, N\}$ and

$$
\begin{aligned}
E_{\beta, \Omega}^{h}\left(\phi^{h}\right):= & \sum_{j=1}^{M-1} \sum_{l=1}^{N-1}\left[\frac{\left|\phi_{j+1, l}^{h}-\phi_{j, l}^{h}\right|^{2}}{2 h_{x}^{2}}+\frac{\left|\phi_{j, l+1}^{h}-\phi_{j, l}^{h}\right|^{2}}{2 h_{y}^{2}}+V_{2}\left(x_{j}, y_{l}\right)\left|\phi_{j, l}^{h}\right|^{2}\right. \\
& \left.+\frac{\beta_{2}}{2}\left|\phi_{j, l}^{h}\right|^{4}+i \Omega\left(\phi^{h}\right)_{j, l}^{*}\left(y_{l} \frac{\phi_{j+1, l}^{h}-\phi_{j-1, l}^{h}}{2 h_{x}}-x_{j} \frac{\phi_{j, l+1}^{h}-\phi_{j, l-1}^{h}}{2 h_{y}}\right)\right] \\
\approx & E_{\beta, \Omega}(\phi),
\end{aligned}
$$

where $\left\{\left(x_{j}, y_{l}\right)\right\}_{j, l}$ are grid points of $\Omega_{\mathbf{x}}$. Table 6.6 lists the numerical energies $E^{h}$ with $\beta_{2}=100$ and $\gamma_{x}=1$ for $\gamma_{y}=1$ and $\gamma_{y}=1.5$, under different rotation speeds $\Omega$ and mesh sizes $h$.

From Table 6.6, we can observe that for $\gamma_{y} \geq 1$ : (i) when $|\Omega|<\gamma_{x y}=1, E^{g}:=$ $\lim _{h \rightarrow 0} E^{h}$ which implies that $\phi_{\beta, \Omega}^{h}$ is a good approximation of the ground state $\phi_{\beta, \Omega}^{g}(\mathbf{x})$; (ii) when $|\Omega|>\gamma^{x y}, \lim _{h \rightarrow 0} E^{h}=-\infty$ which suggests that there is no ground state when $|\Omega|>\gamma^{x y}$ and the steady state solution $\phi^{h}$ is a spurious numerical ground 
(a).
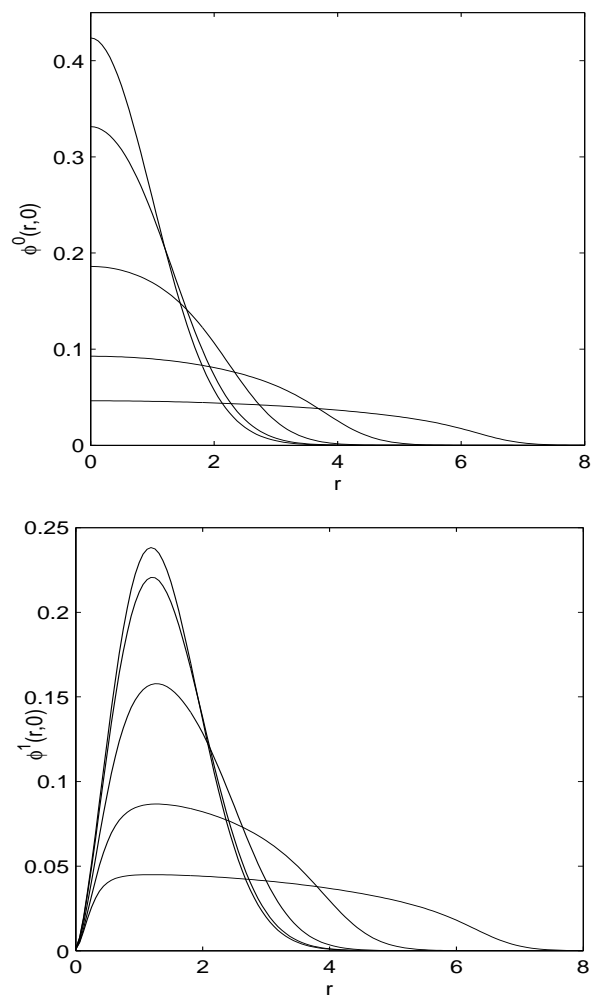

(b).

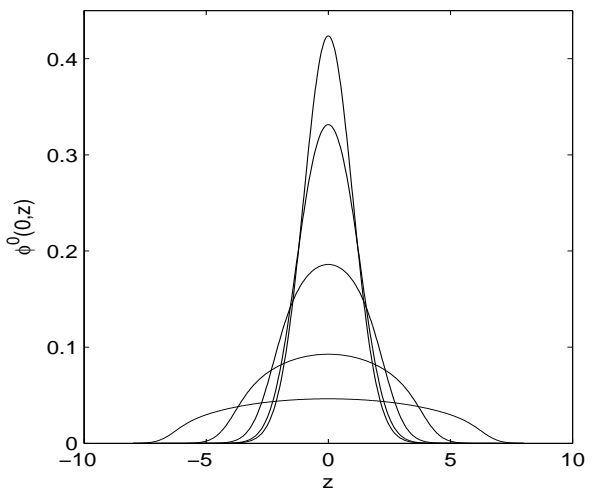

(d).

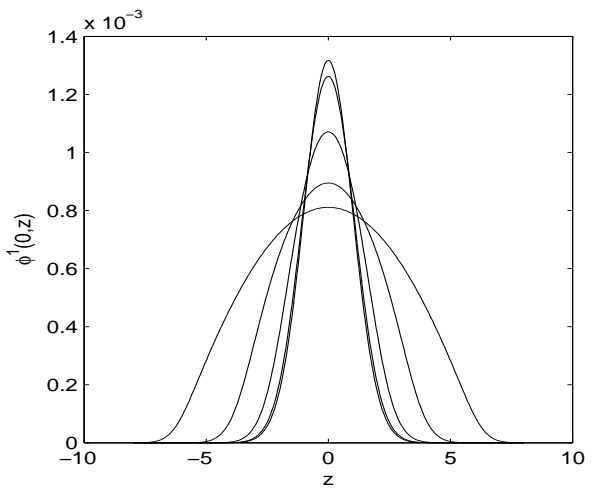

FIG. 6.9. Cylindrically symmetric (upper row) and central vortex (lower row) states in $3 D$ with $\gamma_{x}=\gamma_{y}=\gamma_{z}=1$ for $\beta_{3}=0.0,10.0,100.0,1000.0,10000.0$ (in the order of decreasing of peak) in Example 4. a). $\left.\left.\left.\phi^{0}(r, 0) ; b\right) . \phi^{0}(0, z) ; c\right) \cdot \phi^{1}(r, 0) ; d\right) . \phi^{1}(0, z)$.

a)

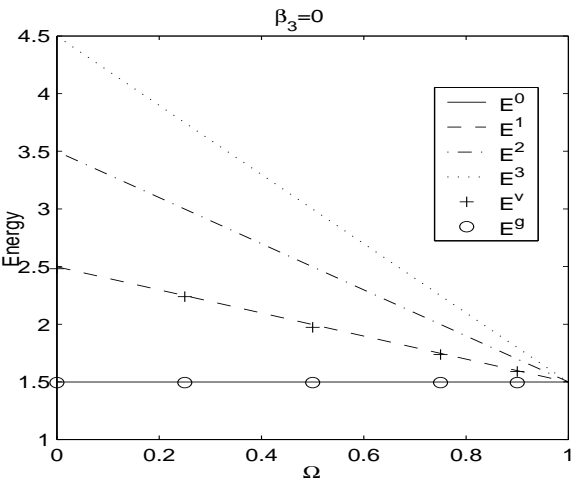

FIG. 6.10. Energy diagrams for ground state, cylindrically symmetric state, central vortex states in $3 D$ with $\gamma_{x}=\gamma_{y}=\gamma_{z}=1.0$ in Example 4 for different $\beta$ and $\Omega$.

state; (iii) when $\gamma_{x y}<|\Omega| \leq \gamma^{x y}$ in an anisotropic trap, our numerical results suggest that there is no ground state. Similar results can be obtained in $3 \mathrm{D}$ from our additional numerical experiments.

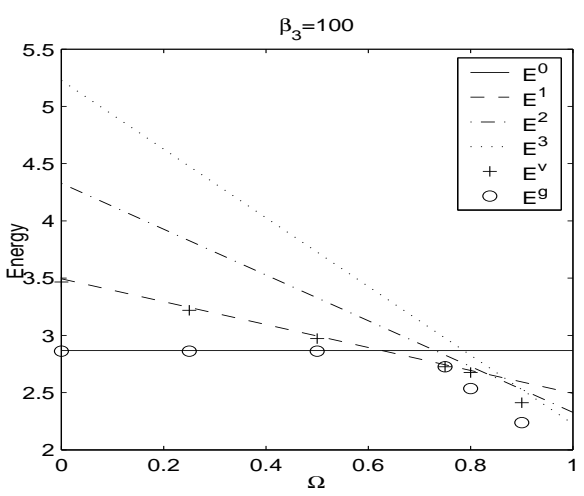

b) 


\begin{tabular}{|c|c|c|c|c|c|c|}
\hline & & $\beta_{2}$ & $\Omega=0$ & $\Omega=0.1$ & $\Omega=0.5$ & $\Omega=0.75$ \\
\hline \multirow[t]{5}{*}{$\max$} & \multirow{5}{*}{$\left|\phi_{\beta, \Omega}^{g}\right|^{2}-\left|\phi_{\beta}^{\mathrm{TF}}\right|^{2} \mid$} & 100 & $3.827 \mathrm{E}-3$ & $3.832 \mathrm{E}-3$ & $5.641 \mathrm{E}-2$ & $4.516 \mathrm{E}-2$ \\
\hline & & 200 & $2.177 \mathrm{E}-3$ & $2.181 \mathrm{E}-3$ & $3.989 \mathrm{E}-2$ & $3.966 \mathrm{E}-2$ \\
\hline & & 400 & $1.2849 \mathrm{E}-3$ & $1.288 \mathrm{E}-3$ & $2.820 \mathrm{E}-2$ & $2.657 \mathrm{E}-2$ \\
\hline & & 800 & $7.125 \mathrm{E}-4$ & $7.407 \mathrm{E}-4$ & $1.358 \mathrm{E}-2$ & $1.659 \mathrm{E}-2$ \\
\hline & & 1000 & $6.157 \mathrm{E}-4$ & $6.172 \mathrm{E}-4$ & $1.161 \mathrm{E}-2$ & $1.321 \mathrm{E}-2$ \\
\hline \multirow{5}{*}{\multicolumn{2}{|c|}{$E_{\beta, \Omega}^{g}-E_{\beta, \Omega}^{\mathrm{TF}}$}} & 100 & 0.1167 & 0.117 & $9.879 \mathrm{E}-2$ & 0.932 \\
\hline & & 200 & $8.950 \mathrm{E}-2$ & $8.919 \mathrm{E}-2$ & 0.190 & 1.652 \\
\hline & & 400 & $6.846 \mathrm{E}-2$ & $6.811 \mathrm{E}-2$ & 0.262 & 2.620 \\
\hline & & 800 & $7.858 \mathrm{E}-2$ & $5.079 \mathrm{E}-2$ & 1.325 & 3.748 \\
\hline & & 1000 & $4.684 \mathrm{E}-2$ & $4.640 \mathrm{E}-2$ & 1.586 & 4.243 \\
\hline \multirow{5}{*}{\multicolumn{2}{|c|}{$\mu_{\beta, \Omega}^{g}-\mu_{\beta, \Omega}^{\mathrm{TF}}$}} & 100 & 0.183 & 0.183 & $9.954 \mathrm{E}-2$ & 0.402 \\
\hline & & 200 & 0.142 & 0.1423 & $2.520 \mathrm{E}-2$ & 0.890 \\
\hline & & 400 & 0.110 & 0.109 & 0.128 & 1.568 \\
\hline & & 800 & $8.643 \mathrm{E}-2$ & $8.342 \mathrm{E}-2$ & 0.719 & 2.441 \\
\hline & & 1000 & $7.649 \mathrm{E}-2$ & $7.650 \mathrm{E}-2$ & 0.870 & 2.796 \\
\hline
\end{tabular}

TABLE 6.5. Errors of the TF approximation in $2 D$.

\begin{tabular}{cccccc}
\hline$\gamma_{y}=1$ & $\Omega$ & $h=1 / 2$ & $h=1 / 4$ & $h=1 / 8$ & $h=1 / 16$ \\
& 0.0 & 3.937 & 3.944 & 3.945 & 3.945 \\
& 0.8 & 3.164 & 3.185 & 3.186 & 3.186 \\
& 1.0 & 2.402 & 1.952 & 1.598 & 1.388 \\
& 1.5 & -1.744 & -13.550 & -37.952 & -52.941 \\
\hline$\gamma_{y}=1.5$ & 0.0 & 4.831 & 4.843 & 4.846 & 4.846 \\
& 0.8 & 4.294 & 4.289 & 4.285 & 4.285 \\
& 1.0 & 3.592 & 3.298 & 2.911 & 2.716 \\
& 1.25 & 2.478 & 0.751 & -1.491 & -2.169 \\
& 1.5 & 1.010 & -4.024 & -10.446 & -12.99 \\
& 2.0 & -3.56 & -17.66 & -39.74 & -53.31 \\
\hline
\end{tabular}

TABLE 6.6. Energy $E^{h}$ in $2 D$ with $\beta_{2}=100$.

\section{Conclusion}

Based on our asymptotic and extensive numerical studies, we can draw the following conjectures for a rotating BEC:

(i) The nonlinear eigenvalue problem (2.12) admits infinitely many eigenfunctions which are linearly independent. When $\beta_{d} \geq 0$ and $|\Omega|<\gamma_{x y}$, if the eigenfunctions of (2.12) are ranked according to their energies, $\phi^{g}, \phi^{1}, \ldots$, then the corresponding eigenvalues (or chemical potentials ) are in the same order, i.e.

$$
E_{\beta, \Omega}\left(\phi^{g}\right) \leq E_{\beta, \Omega}\left(\phi^{1}\right) \leq E_{\beta, \Omega}\left(\phi^{2}\right) \leq \ldots \Longrightarrow \mu_{\beta, \Omega}\left(\phi^{g}\right) \leq \mu_{\beta, \Omega}\left(\phi^{1}\right) \leq \mu_{\beta, \Omega}\left(\phi^{2}\right) \leq \ldots .
$$

(ii) For any fixed $\beta_{d} \geq 0$, there exists a critical angular frequency $0<\Omega^{c} \leq \gamma_{x y}$ such that when $\Omega>\Omega^{c}$ the ground state breaks symmetry, i.e. quantized vortices appear in the ground state. When $\beta_{d}$ increases, $\Omega^{c}$ increases too. Similar conclusions are valid for the central vortex ground state. Furthermore, we have

$$
\Omega^{c} \approx E_{\beta, 0}^{1}-E_{\beta, 0}^{0}, \quad \Omega^{v} \approx E_{\beta, 0}^{2}-E_{\beta, 0}^{1} .
$$


(iii) For any fixed $|\Omega|<\gamma_{x y}$, the energy and chemical potential of any stationary state increase when $\beta_{d}$ increases. Furthermore, when $\beta_{d} \rightarrow \infty$, they have the following asymptotics:

$$
E_{\beta, \Omega}^{s}=O\left(\beta^{2 /(2+d)}\right), \mu_{\beta, \Omega}^{s}=O\left(\beta^{2 /(2+d)}\right), \text { when } \beta \rightarrow \infty, \quad s=g, v, 0,1, \ldots,
$$

and the ratios between them are constants, i.e.

$$
\lim _{\beta_{d} \rightarrow \infty} \frac{E_{\beta, \Omega}^{v}}{E_{\beta, \Omega}^{g}}=\lim _{\beta_{d} \rightarrow \infty} \frac{\mu_{\beta, \Omega}^{v}}{\mu_{\beta, \Omega}^{g}}=1, \lim _{\beta_{d} \rightarrow \infty} \frac{E_{\beta, \Omega}^{s}}{E_{\beta, \Omega}^{g}}=\text { const, } \lim _{\beta_{d} \rightarrow \infty} \frac{\mu_{\beta, \Omega}^{s}}{\mu_{\beta, \Omega}^{g}}=\text { const, } s \geq 0 .
$$

(iv) For any fixed $\beta_{d} \geq 0$, the energy and chemical potential of any stationary state decrease when $\Omega$ increases from 0 to $\gamma_{x y}$ or decreases from 0 to $-\gamma_{x y}$. Furthermore, the energy of the ground state has the following asymptotic when $\Omega$ is near $\pm \gamma_{x y}$ :

$$
E_{\beta, \Omega}^{g}=E_{\beta, \pm \gamma_{x y}^{\mp}}^{g}+O\left(\gamma_{x y} \mp \Omega\right), \quad \Omega \rightarrow \pm \gamma_{x y}^{\mp} .
$$

(v) In disk-shaped condensation, i.e. $\gamma_{x}=O(1), \gamma_{y}=O(1)$ and $\gamma_{z} \gg 1$, the ground state and its energy and chemical potential in $3 \mathrm{D}$ with interaction $\beta$ can be well approximated by those of its $2 \mathrm{D}$ reduction with interaction $\beta_{2}=\beta \sqrt{\gamma_{z} / 2 \pi}$.

(vi) In the semiclassical regime, the TF approximation is very accurate for the density of the ground state except at the vortex core; where the TF approximate energy and chemical potential converge to the ground state energy and chemical potential respectively only when $|\Omega| \approx 0$, but diverge when $|\Omega|$ is near $\gamma_{x y}$.

Acknowledgment. W.B. acknowledges support by the National University of Singapore grant No. R-151-000-030-112. P.A.M. acknowledges support from his WITTGENSTEIN-AWARD 2000 funded by the Austrian National Science Fund FWF.

\section{REFERENCES}

[1] A. Aftalion and Q. Du, Vortices in a rotating Bose-Einstein condensate: Critical angular velocities and energy diagrams in the Thomas-Fermi regime, Phys. Rev. A, 64, 063603 2001.

[2] A. Aftalion and T. Riviere, Vortex energy and vortex bending for a rotating Bose-Einstein condensate, Phys. Rev. A 64, 043611, 2001.

[3] A. Aftalion and I. Danaila, Three-dimensional vortex configurations in a rotating Bose Einstein condensate, Phys. Rev. A 68, 023603, 2003.

[4] M. H. Anderson, J.R. Ensher, M.R. Matthews, C.E. Wieman and E.A. Cornell, Observation of Bose-Einstein condensation in a dilute atomic vapor, Science, 269, 198, 1995.

[5] W. Bao, Ground states and dynamics of multi-component Bose-Einstein condensates, SIAM Multiscale Modeling and Simulation, 2, 210-236, 2004.

[6] W. Bao and Q. Du, Computing the ground state solution of Bose-Einstein condensates by a normalized gradient flow, SIAM J. Sci. Comput., 25, 1674-1697, 2004.

[7] W. Bao, D. Jaksch and P.A. Markowich, Numerical solution of the Gross-Pitaevskii Equation for Bose-Einstein condensation, J. Comput. Phys., 187, 318 - 342, 2003.

[8] W. Bao and W. Tang, Ground state solution of trapped interacting Bose-Einstein condensate by directly minimizing the energy functional, J. Comput. Phys., 187, 230-254, 2003.

[9] W. Bao and Y. Zhang, Dynamics of the ground state and central vortec states in Bose-Einstein condensation, Math. Mod. Meth. Appl. Sci., to appear.

[10] C. C. Bradley, C.A. Sackett and R.G. Hulet, Evidence of Bose-Einstein condensation in an atomic gas with attractive interactions, Phys. Rev. Lett. 75, 1687, 1995.

[11] Y. Castin and R. Dum, Bose-Einstein condensates with vortices in rotating traps, Eur. Phys. J. D 7, 399-412, 1999 
[12] B. M. Caradoc-Davis, R. J. Ballagh and P. B. Blakie, Three-dimensional vortex dynamics in Bose-Einstein condensates, Phys. Rev. A 62, 011602, 2000

[13] B. M. Caradoc-Davis, R. J. Ballagh and P. B. Blakie, Coherent dynamics of vortex formation in trapped Bose-Einstein condensates, Phys. Rev. Lett. 83, 895, 1999.

[14] S. -M. Chang, W. -W. Lin and S. -F. Shieh, Gauss-Seidel-type methods for energy states of a multi-component Bose-Einstein condensate, J. Comput. Phys., to appear.

[15] M. L. Chiofalo, S. Succi and M.P. Tosi, Ground state of trapped interacting Bose-Einstein condensates by an explicit imaginary-time algorithm, Phys. Rev. E 62, 7438, 2000.

[16] F. Dalfovo and S. Giorgini, Theory of Bose-Einstein condensation in trapped gases, Rev. Mod. Phys. 71, 463, 1999.

[17] K. B. Davis, M.O. Mewes, M.R. Andrews, N.J. van Druten, D.S. Durfee, D.M. Kurn and W. Ketterle, Bose-Einstein condensation in a gas of sodium atoms, Phys. Rev. Lett. 75 , 3969, 1995

[18] R. J. Donnelly, Quantizied Vortices in Helium II, Cambridge University Press, 1991

[19] J. R. Ensher, D. S. Jin, M. R. Matthews, C. E. Wieman and E. A. Cornell, Bose-Einstein condensation in a dilute gas: measurement of energy and ground-state occupation, Phys. Rev. Lett. 77, 4984, 1996.

[20] D. L. Feder, C. W. Clark and B. I. Schneider, Nucleation of vortex arrays in rotating anisotropic Bose-Einstein condensates, Phys. Rev. A 61, 011601, 1999.

[21] D. L. Feder, C. W. Clark and B. I. Schneider, Vortex stability of interacting Bose-Einstein condensates confined in anisotropic harmonic traps, Phys. Rev. Lett. 82, 4956, 1999.

[22] A. L. Fetter and A. A. Svidzinsky, Vortices in a trapped dilute Bose- Einstein condensate, J. Phys. Condens. Matter 13, R135-194, 2001.

[23] J.J. Garcia-Ripoll and V.M. Perez-Garcia, Vortex bending and tightly packed vortex latices in Bose-Einstein condensates, Phys. Rev. A, to appear.

[24] J. J. Garcia-Ripoll and V. M. Perez-Garcia, Stability of vortices in inhomogeneous Bose Einstein condensates subject to rotation: a three dimensional analysis, Phys. Rev. A, 60, 4864-4874, 1999.

[25] J. J. Garcia-Ripoll and V. M. Perez-Garcia, Optimizing schrodinger functionals using sobolev gradients: application to quantum mechanics and nonlinear optics, SIAM J. Sci. Comput. $23,1316-1334,2001$.

[26] J.J. Garcia-Ripoll and V. M. Perez-Garcia, Vortex nucleation and hysteresis phenomena in rotating Bose-Einstein condensate, Phys. Rev. A63, 041603, 2001.

[27] B. Jackson, J. F. McCann and C. S. Adams, Vortex formation in dilute inhomogeneous BoseEinstein condensates, Phys. Rev. Lett. 80, 3903, 1998.

[28] B. Jackson, J. F. McCann and C. S. Adams, Vortex line and ring dynamics in trapped BoseEinstein condensates, Phys. Rev. A 61, 013604, 1999.

[29] K. Kasamatsu and M. Tsubota, Nonlinear dynamics for vortex formation in a rotating BoseEinstein condensate, Phys. Rev. A 67, 033610, 2003.

[30] L. Laudau and E. Lifschitz, Quantum Mechanics: Non-relativistic Theory, Pergamon Press, New York, 1977

[31] E. H. Lieb, R. Seiringer and J. Yngvason, Bosons in a trap: a rigorous derivation of the Gross-Pitaevskii energy functional, Phys. Rev. A 61, 3602, 2000.

[32] E. Lundh, C.J. Pethick and H. Smith, Vortices in Bose-Einstein condensated atomic clouds, Phys. Rev. A, 58, 4816-4823, 1998.

[33] K. W. Madison, F. Chevy, W. Wohlleben and J. Dal- ibard, Vortex formation in a stirred Bose-Einstein condensate, Phys. Rev. Lett. 84, 806, 2000.

[34] M. R. Matthews, B. P. Anderson, P. C. Haljan, D. S. Hall, C. E. Wieman and E. A. Cornell, Vortices in a Bose-Einstein condensate, Phys. Rev. Lett. 83, 2498, 1999.

[35] M. Modugno, L. Pricoupenko and Y. Castin, Bose-Einstein condensates with a bent vortex in rotating traps, Eur. Phys. J. D, 22, 235-257, 2003.

[36] L. P. Pitaevskii, Vortex lines in a imperfect Bose gase, Zh. Eksp. Teor. Fiz. 40, 646, 1961. (Sov. Phys. JETP 13, 451, 1961).

[37] L. Pitaevskii and S. Stringari, Bose-Einstein Condensation, Oxford, New York, Clarendon Press, 2003.

[38] C. Raman, J. R. Abo-Shaeer, J. M. Vogels, K. Xu and W. Ketterle, Vortex nucleation in a stirred Bose-Einstein condensate, Phys. Rev. Lett. 87, 210402, 2001.

[39] P. Rosenbuch, V. Bretin and J. Dalibard, Phys. Rev. lett., 89, 200403, 2002.

[40] R. Seiringer, Gross-Pitaevskii theory of the rotating Bose gas, Comm. Math. Phys. 229, 491509, 2002.

[41] R. Seiringer, Ground state asymptotics of a dilute rotating Gas, J. Phys. A: Math. Gen. 36, 9755-9778, 2003 
[42] A. A. Svidzinsky and A. L. Fetter, Dynamics of a vortex in a trapped Bose-Einstein condensate, Phys. Rev. A 62, 063617, 2000.

[43] L. Simon, Asymptotics for a class of nonlinear evolution equations, with applications to geometric problems, Ann. Math., 118, 525-571, 1983.

[44] S. Sinha and Y. Castin, Dynamic instability of a rotating Bose-Einstein condensate, Phys. Rev. Lett., 87, 190402, 2001.

[45] M. Tsubota, K. Kasamatsu and M. Ueda, Vortex lattice formation in a rotating Bose-Einstein condensate, Phys. Rev. A, 65, 023603, 2000.

[46] A.H. Zhou, An analysis of finite-dimensional approximations for the ground state solution of Bose-Einstein condensates, Nonlinearity 17, 541-550, 2004. 